\title{
Skeleton-secreted PDGF-BB mediates arterial stiffening
}

\author{
Lakshmi Santhanam, ${ }^{1,2,3}$ Guanqiao Liu, ${ }^{4,5}$ Sandeep Jandu, ${ }^{1}$ Weiping Su, ${ }^{4,6}$ Bulouere P. Wodu, ${ }^{7}$ William Savage, ${ }^{3}$ Alan Poe, ${ }^{2}$ \\ Xiaonan Liu, ${ }^{4,5}$ Lacy M. Alexander, ${ }^{8}$ Xu Cao, ${ }^{4}$ and Mei Wan ${ }^{4}$
}

DDepartment of Anesthesiology and Critical Care Medicine and ${ }^{2}$ Department of Biomedical Engineering, The Johns Hopkins University School of Medicine, Baltimore, Maryland, USA. ${ }^{3}$ Department of Chemical and Biomolecular Engineering, Whiting School of Engineering, The Johns Hopkins University, Baltimore, Maryland, USA. ${ }^{4}$ Department of Orthopaedic Surgery, The Johns Hopkins University School of Medicine, Baltimore, Maryland, USA. ${ }^{5}$ Department of Orthopaedics and Traumatology, Nanfang Hospital, Southern Medical University, Guangzhou, Guangdong, China. ${ }^{6}$ Department of Orthopaedic Surgery, The Xiangya Hospital of Central South University, Changsha, Hunan, China. ${ }^{7}$ Department of Biotechnology, The Johns Hopkins University, Baltimore, Maryland, USA. ${ }^{8}$ Department of Kinesiology, Penn State University, University Park, Pennsylvania, USA.

\begin{abstract}
Evidence links osteoporosis and cardiovascular disease but the cellular and molecular mechanisms are unclear. Here we identify skeleton-secreted platelet-derived growth factor-BB (PDCF-BB) as a key mediator of arterial stiffening in response to aging and metabolic stress. Aged mice and those fed high-fat diet (HFD), relative to young mice and those fed normal chow food diet, respectively, had higher serum PDCF-BB and developed bone loss and arterial stiffening. Bone/bone marrow preosteoclasts in aged mice and HFD mice secrete an excessive amount of PDGF-BB, contributing to the elevated PDGF-BB in blood circulation. Conditioned medium prepared from preosteoclasts stimulated proliferation and migration of the vascular smooth muscle cells. Conditional transgenic mice, in which PDCF-BB is overexpressed in preosteoclasts, had 3-fold higher serum PDGF-BB concentration and developed simultaneous bone loss and arterial stiffening spontaneously at a young age. Conversely, in conditional knockout mice, in which PDCF-BB is deleted selectively in preosteoclasts, HFD did not affect serum PDGF-BB concentration; as a result, HFD-induced bone loss and arterial stiffening were attenuated. These studies confirm that preosteoclasts are a main source of excessive PDCF-BB in blood circulation during aging and metabolic stress and establish the role of skeleton-derived PDCF-BB as an important mediator of vascular stiffening.
\end{abstract}

\section{Introduction}

Accumulating evidence supports a link between bone metabolism and the vascular system. Cross-sectional and longitudinal studies have shown a direct association between osteoporosis and cardiovascular disease (CVD) (1-5), 2 primary conditions that cause substantial morbidity and death in older people. In fact, the correlation between these 2 disorders is independent of age. Particularly, bone mineral density (BMD) is inversely and independently correlated with atherosclerosis and its established marker, aortic calcification (6-10). Low BMD has been associated with cardiovascular morbidity and mortality. Although the concept of a bone/vascular axis has long been proposed $(3,4,11)$, the exact cellular and molecular basis for the interplay between the skeletal and vascular systems is poorly understood. Several hypotheses have been proposed to explain the link between osteoporosis and CVD, including shared risk factors, common pathological mechanisms and genetic factors, and a causal association (12). However, high bone turnover is associated with cardiovascular death in the elderly, independent of sex and overall health (13).

\section{Related Commentary: https://doi.org/10.1172/JCl153644}

Authorship note: LS, GL, and SJ contributed equally to this work.

Conflict of interest: The authors have declared that no conflict of interest exists. Copyright: (5) 2021, American Society for Clinical Investigation.

Submitted: December 23, 2020; Accepted: August 24, 2021; Published: October 15, 2021.

Reference information: J Clin Invest. 2021;131(20):e147116.

https://doi.org/10.1172/JCl147116.
Bisphosphonate therapy for osteoporosis decreases the risk of aortic valve and thoracic aorta calcification (14). These findings strongly suggest that bone-derived cues may directly affect the vascular system. Furthermore, accumulating clinical studies have demonstrated an association between low bone mass and vascular calcification (15-17), a well-defined independent risk factor for CVD and mortality. Vascular calcification and bone mineralization are both actively regulated processes that may share common pathogenetic mechanisms. Multiple factors including modified low-density lipoprotein (LDL), inflammatory cytokines, Wnt signaling, bone morphogenetic proteins, matrix proteins (such as thrombospondin, tenascin, osteopontin, osteocalcin, osteoprotegerin, matrix Gla protein, cathepsins, and DMP-1), parathyroid hormone, phosphate, and vitamins $\mathrm{D}$ and $\mathrm{K}$ are implicated in both bone and vascular metabolism, suggesting the interaction of these 2 pathological conditions.

The skeleton is not only a recipient for hormonal input but also an endocrine organ that regulates the homeostasis of peripheral organs $(18,19)$. For example, osteoclasts regulate the activity of other cells by secreting "clastokines" or releasing factors from bone matrix via bone resorption (20-23). Osteoclasts are multinucleated cells that have the ability to degrade mineralized matrices, such as bone and calcified cartilage. Osteoclasts in adults are derived mainly from bone marrow monocytes/macrophages (Mo/Macs). During osteoclastogenesis, Mo/Macs sequentially express colony-stimulating factor-1 receptor followed by receptor activator of nuclear factor $\kappa \mathrm{B}$ (RANK) and tartrate-resistant acid phosphatase (TRAP) in response to stimulation with macrophage 
A

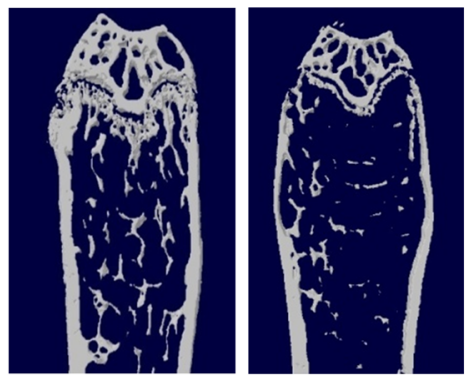

$\mathbf{F}$

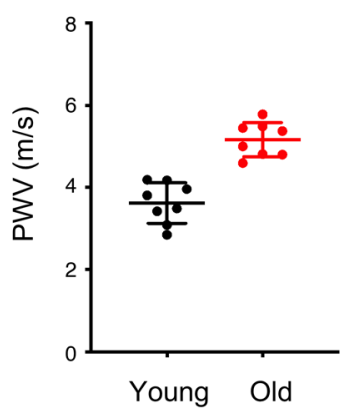

H

$\mathrm{CHD}$
B

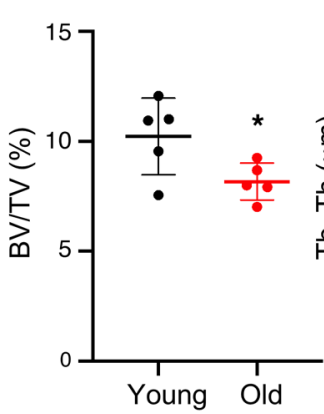

G

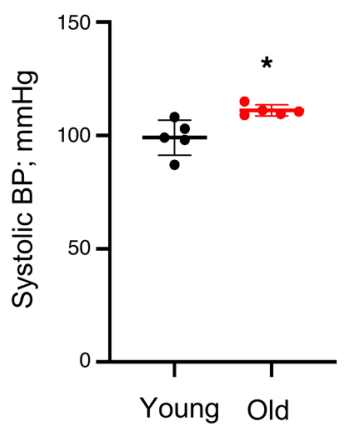

I

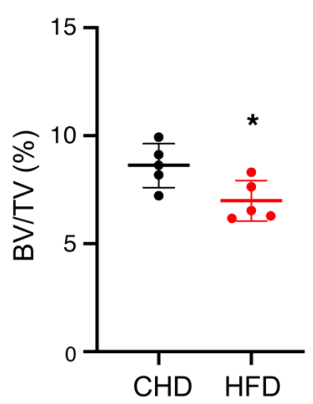

$\mathbf{N}$

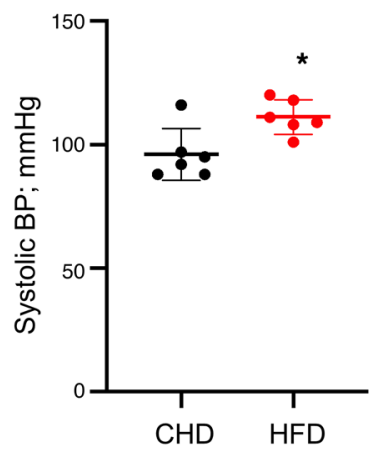

C

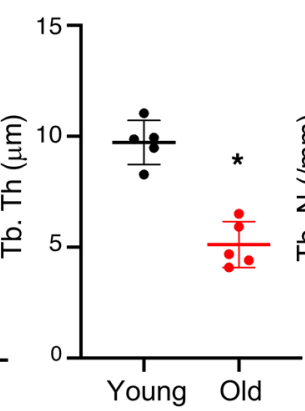

D

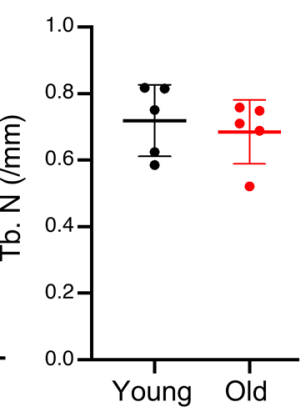

E

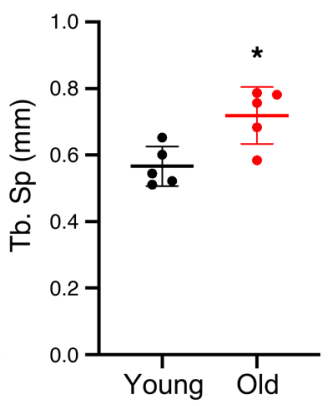

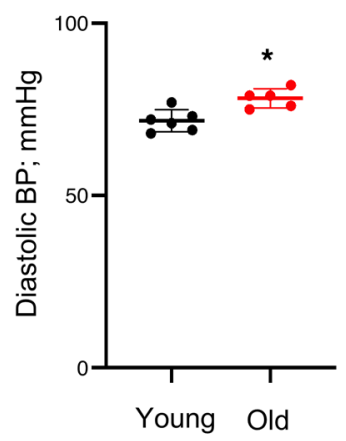

K

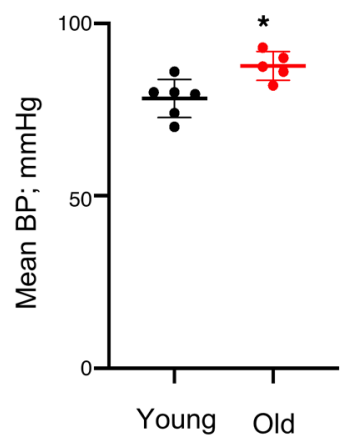

$\mathbf{L}$

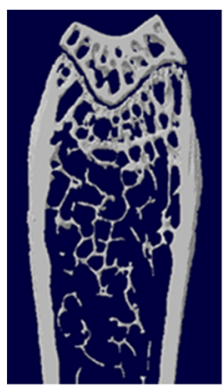

M
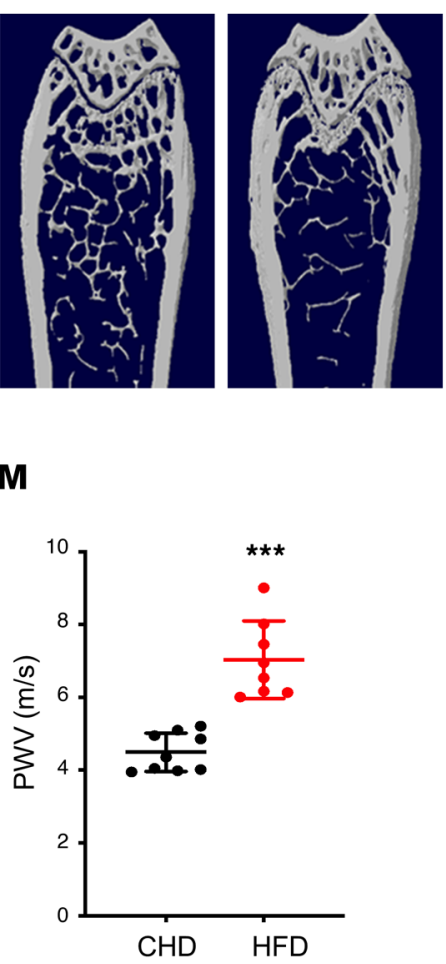

J

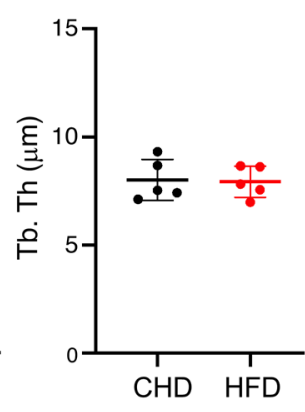

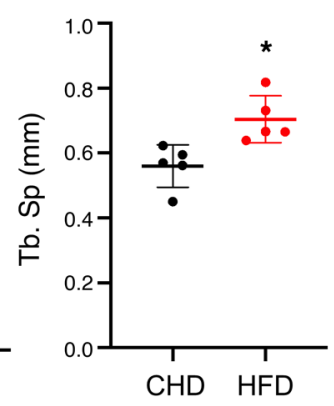

Figure 1. Aged mice and HFD-challenged mice develop low bone mass and an arterial stiffening phenotype. (A-E) Representative $\mu \mathrm{CT}$ images (A) and quantitative analysis (B-E) of the trabecular bone area of the distal femur from 4- and 20-month-old male C57BL/6 mice. Bone volume per tissue volume (BV/TV) (B), trabecular bone thickness (Tb.Th) (C), trabecular bone number (Tb.N) (D), and trabecular bone separation (Tb.Sp) (E). (F and G) Pulse-wave velocity (PWV) and systolic, diastolic, and mean blood pressure (BP) measurements of 4- and 20-month-old male mice. (H-L) Representative $\mu C T$ images (H) and quantitative analysis (I-L) of the trabecular bone area of the distal femur from 3-month-old male C57BL/6 mice fed a Western HFD or normal CHD for 5 months. BV/TV (I), Tb.Th (J), Tb.N (K), and Tb.Sp (L). (M and $\mathbf{N}$ ) PWV and BP measurements of the mice fed HFD or CHD. $n=5$ to 9 . Data are mean \pm $\mathrm{SD},{ }^{*} P<0.05,{ }^{*} P<0.01,{ }^{* * *} P<0.005$, as determined by Student's $t$ tests. 
colony-stimulating factor (M-CSF) and RANK ligand. Eventually, mononuclear preosteoclasts fuse to form multinuclear osteoclasts $(20,24,25)$. Osteoclast lineage cells normally have a much shorter life span ( 2 weeks) relative to osteoblasts ( 3 months) and other bone cells (26). After osteoclasts have eroded bone to a particular depth from the surface, they die quickly. During estrogen deficiency or aging, the life span of this lineage of cells is prolonged through an antiapoptosis mechanism $(26,27)$, resulting in increased bone resorption. It has been demonstrated that bone/ bone marrow mononuclear preosteoclasts secrete platelet-derived growth factor-BB (PDGF-BB) to maintain normal bone homeostasis in healthy, young mice (28), whereas abnormally high production of PDGF-BB from preosteoclasts leads to skeletal disorders, such as osteoarthritis (29).

With advancing age, complex structural and functional changes occur in the arterial system. The large compliance vessels, including the aorta and its major branches, stiffen with age, and this stiffening can be accelerated by comorbidities, including obesity and atherosclerosis (30-35). Increased aortic stiffness increases central arterial pressure and pulse pressure and is an independent risk factor for cardiovascular morbidity and death (36-41). Moreover, arterial aging is characterized by accelerated development of atherosclerotic lesions and neointima formation during atherosclerosis (42). Hallmarks of the stiff vessel include intimal and medial thickening and an increased collagen/elastin ratio in the arterial wall, as well as elastin fracture (43-46). Traditionally, it was suggested that the remodeling and accumulation of the vascular matrix is the main element of vascular stiffening. However, recent studies have recognized that vascular smooth muscle cell (VSMC) dysfunction and stiffening are major contributors to vascular stiffening (47-49). Thus, augmented VSMC motility, proliferation, and dedifferentiation are critical to vascular stiffening.

PDGFs are important serum factors that stimulate smooth muscle cell migration and proliferation $(50,51)$. The PDGF family consists of 5 members: PDGF-AA, PDGF-BB, PDGF-CC, PDGF$\mathrm{DD}$, and PDGF-AB. Genetic manipulations combined with various inhibitory strategies have provided strong evidence for the prominent role of PDGF-BB in the development of neointimal hyperplasia after injury and in atherosclerosis (52-56). Although PDGF and its receptors are detected in many cultured vascular cells and in arteries after injury, PDGF-BB is expressed at very low or undetectable levels in normal vessels (52). Increased expression of PDGF receptors was detected in VSMCs of aged arteries (57). Serum PDGF levels increase in hypertension (58) and hypercholesterolemia (59). Thus, PDGF-BB likely serves as a vascular aging-inducing factor.

In the current study, we aimed to determine whether circulating PDGF-BB is elevated in arterial stiffening associated with aging and high-fat diet (HFD) and whether or how bone/bone marrow preosteoclasts are involved in this process. In aged mice and mice fed a Western HFD, mononuclear preosteoclasts in bone/bone marrow produced markedly more PDGF-BB relative to young mice and mice fed a chow-food diet (CHD), respectively. We generated conditional knockout and transgenic mice, in which PDGF-BB was deleted and overexpressed, respectively, in $\mathrm{TRAP}^{+}$preosteoclasts, and found that preosteoclast-derived PDGF-BB was both sufficient and required for HFD-induced augmented arterial stiffness.

\section{Results}

Animals develop low bone mass and an arterial stiffening phenotype in response to aging and HFD challenge. We first assessed the changes in bone mass and arterial stiffness in mice with advancing age. Twenty-month-old C57B/L6 mice had a low bone mass phenotype relative to young mice (4 months of age), as detected by $\mu \mathrm{CT}$ analysis (Figure 1A). Although the difference in trabecular number (Tb.N) in aged versus young mice was not significant (Figure 1D), the differences in the remaining 3 parameters were significant. Bone volume per tissue volume (BV/TV; Figure 1B) and trabecular thickness (Tb.Th; Figure 1C) were less, and trabecular space (Tb.Sp; Figure 1E) was greater in 20-month-old mice compared with 4-month-old mice. We also measured blood pressure (BP) and pulse-wave velocity (PWV), an index of in vivo vascular stiffness. PWV was significantly higher in 20-month-old mice than in 4-month-old mice (Figure 1F). Consistently, systolic, diastolic, and mean BPs of the old mice were all higher than those of the young mice (Figure $1 G$ ).

Next, to observe accelerated deterioration of the bone and vasculature, we used a HFD challenge, because a HFD induces bone loss and increases aortic stiffness and endothelial dysfunction in mice (10, 60-62). Baseline PWV was measured, after which we placed mice on a HFD. We then examined the phenotypic changes of bone and vasculature in HFD-challenged and control mice. $\mu \mathrm{CT}$ analysis showed less bone mass (Figure 1H), lower BV/TV (Figure 1I), lower Tb.N (Figure 1K), and greater Tb.Sp (Figure 1L) in HFD mice compared with CHD mice. The difference in Tb.Th between groups was not significant (Figure 1J). Greater arterial stiffness was also observed in HFD mice relative to CHD mice, as indicated by higher PWV (Figure 1M) and BPs (Figure 1N). Therefore, simultaneous bone loss and arterial stiffening occur with advancing age and under HFD challenge.

Aging mice, rats, and humans and mice with HFD challenge have elevated serum PDGF-BB concentration. Because PDGF-BB has been implicated in the fibrosis of organs $(50,63-65)$ and the modulation of extracellular matrix of the arteries (66-68), we tested the possible involvement of PDGF-BB in regulating arterial stiffness in our model. We measured the change in serum PDGF-BB concentration in aged animals and human subjects. A markedly higher level of serum PDGF-BB was detected in 20-month-old mice versus 3-month-old mice (Figure 2A) and in 25-month-old rats versus 4 -month-old rats (Figure $2 \mathrm{~B}$ ). To determine the potential translational relevance, we measured serum PDGF-BB levels in young and aged human subjects and found higher levels of serum PDGF-BB in aged subjects compared with young subjects (Figure 2C). In addition, serum PDGF-BB concentration was elevated in HFD-fed mice (Figure 2D). These results suggest that PDGF-BB may be associated with age- and diet-induced arterial stiffness.

Bone/bone marrow preosteoclasts are a main source of elevated $P D G F-B B$ in response to aging and HFD challenge. We previously showed that mononuclear $\mathrm{TRAP}^{+}$preosteoclasts are a primary cell type in bone/bone marrow-secreting PDGF-BB (25). To determine whether PDGF-BB production in bone/bone marrow changes with age, we detected PDGF-BB protein expression by immunofluorescence staining of frozen femoral bone tissue sections. Consistent with our previous study (28), $\mathrm{PDGF}^{-B^{+}}$cells were detected in bone/bone marrow of young (3-month-old) mice 
A

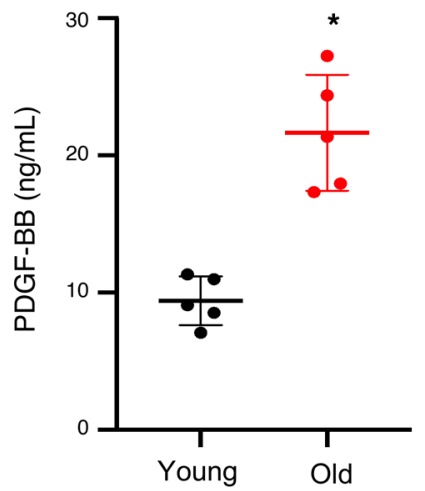

D

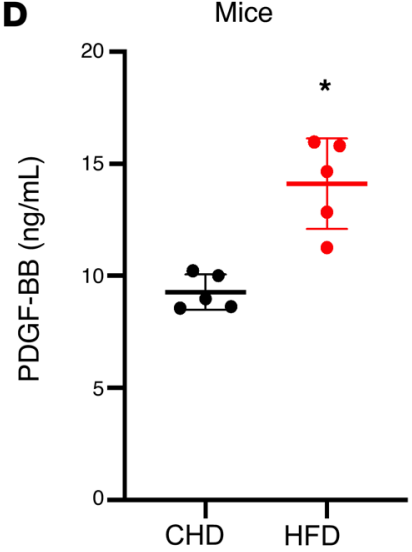

B

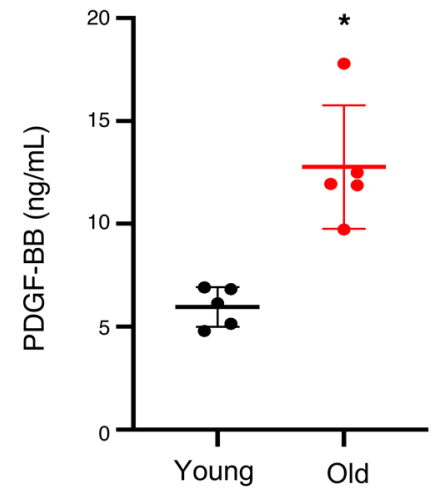

C Humans

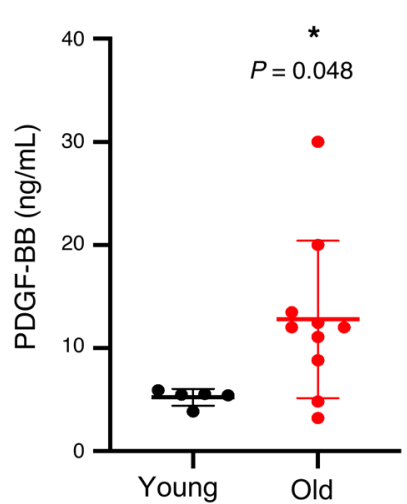

Figure 2. Aged mice, rats, and humans and HFD-challenged mice have elevated serum PDGF-BB concentration. (A) ELISA measurement of serum PDGF-BB concentrations in 3- and 20-month-old mice. (B) ELISA measurement of serum PDGF-BB concentrations in 4- and 25-month-old rats. (C) ELISA measurement of serum PDGF-BB concentrations in young and aged humans. Old, ages 58 to 71 years; young, ages 21 to 26 years. (D) ELISA measurement of serum PDGF-BB concentrations in HFD mice and CHD mice. $n=5$ to 10. Data are mean $\pm \mathrm{SD},{ }^{*} P<0.05$, as determined by Student's $t$ tests.

(Figure 3A). The number of PDGF-BB-expressing cells markedly increased in the bone/bone marrow of 20-month-old mice relative to 3-month-old mice (Figure 3, A and B). PDGF-BB ${ }^{+}$cells were not detected in aorta tissue in either 3- or 20-month-old mice.

To determine whether the increased PDGF-BB is produced by preosteoclasts in bone/bone marrow, we generated TRAP/tdTom mice, in which tdTomato is expressed under TRAP-cre. Therefore, $\mathrm{TRAP}^{+}$preosteoclasts and their descendants are labeled by tdTom fluorescence in the mice. $\mathrm{tdTom}^{+}$cells were abundant in bone tissue, and the majority of PDGF-BB-expressing cells are tdTom ${ }^{+}$ cells (Figure $3 \mathrm{C}$ ). We did detect a few $\mathrm{tdTom}^{+}$cells in the aorta tissue. However, none of the cells in the aorta expressed PDGF-BB (Figure 3D). These results suggest that although there is a nonspecific Cre expression, cells in aortic tissue do not produce PDGFBB. Therefore, the effect of local aorta tissue-produced PDGF-BB can be excluded by using this TRAP-Cre line.

Moreover, we performed FACS sorting to isolate the TRAP/ tdTom $^{+}$cells from femoral bone/bone marrow cells (Figure 3E) and conducted real-time qPCR analysis. TRAP/tdTom ${ }^{+}$cells isolated from HFD-challenged mice had much higher Pdgfb expression compared with those from CHD mice (Figure 3F). To further validate the abnormally high expression of $P d g f b$ in the osteoclast precursors, we detected Pdgfb expression in bone/bone marrow $\mathrm{RANK}^{+}$cells with exclusion of the CD3/B220/Ter119+ cells (the sum of $\mathrm{T}$ cells, B cells, and erythrocytes). We detected markedly greater expression of Pdgfb in CD3/B220/Ter119- RANK $^{+}$osteo- clast precursors from 20-month-old mice versus 6-month-old mice (Figure 3G) and HFD mice versus CHD mice (Figure 3H). Together, the results suggest that bone/bone marrow preosteoclasts secrete excessive PDGF-BB in response to aging or HFD challenge.

Preosteoclast-derived PDGF-BB stimulates VSMC proliferation and migration. One of the important functions of PDGF-BB is to stimulate proliferation and migration of VSMCs, favoring pathological vascular remodeling and arterial stiffening $(66,67,69,70)$. We investigated whether preosteoclast-secreted PDGF-BB is sufficient to induce phenotypic change of VSMCs using conditioned media (CM) of preosteoclast cultures. Bone marrow Mo/Macs isolated from mice differentiate into $\mathrm{TRAP}^{+}$mononuclear preosteoclasts 3 days after treatment with M-CSF and RANK ligand, and most cells differentiate into $\mathrm{TRAP}^{+}$multinuclear mature osteoclasts 7 days after treatment (Figure 4A). We collected CM from cells at 0, 3, and 8 days of M-CSF and RANK ligand treatment, which represent $\mathrm{Mo} / \mathrm{Mac} \mathrm{CM}$, preosteoclast CM, and osteoclast CM, respectively. Dramatically elevated PDGF-BB concentration was detected in preosteoclast $\mathrm{CM}$ relative to $\mathrm{Mo} / \mathrm{Mac} \mathrm{CM}$, whereas PDGF-BB concentration in osteoclast $\mathrm{CM}$ was lower compared with preosteoclast CM (Figure 4B). Importantly, rat VSMCs showed increased proliferation (Figure 4C) and migration (Figure 4D) when the cells were incubated with preosteoclast $\mathrm{CM}$ relative to the cells with $\mathrm{Mo} /$ Mac CM. These effects of preosteoclast CM were antagonized by PDGF-BB-neutralizing antibody. Therefore, PDGF-BB secreted by preosteoclasts can stimulate VSMC proliferation and migration. 
A
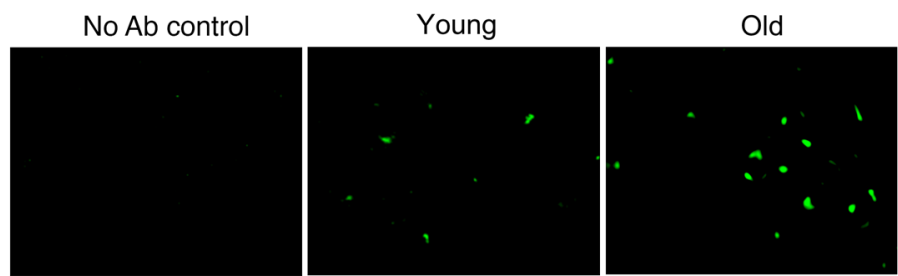

Aorta
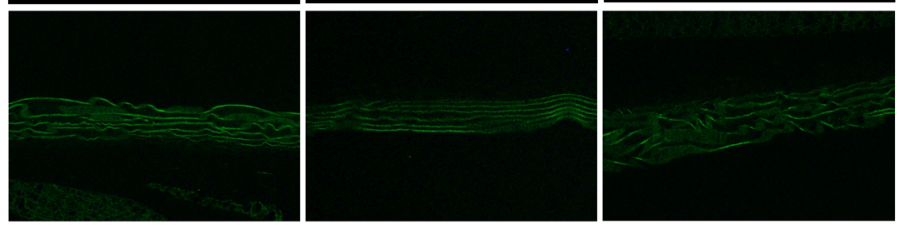

B

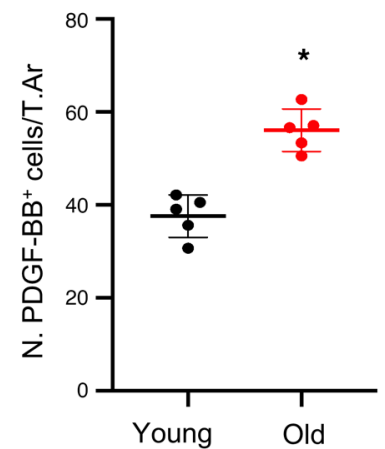

c

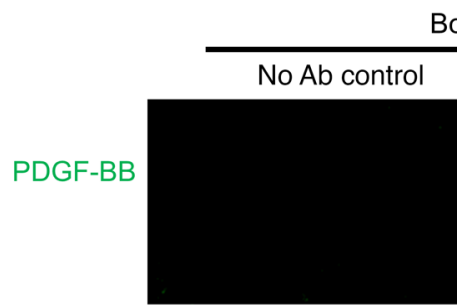

tdTom

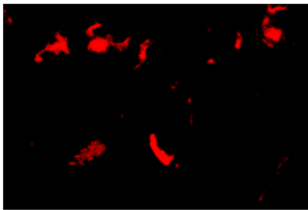

PDGF-BB/tdTom/

DAPI
Bone
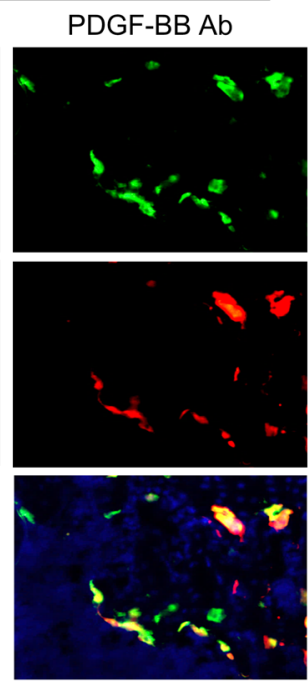

D

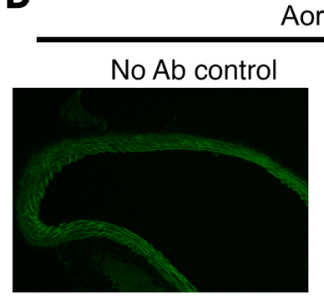

Aorta
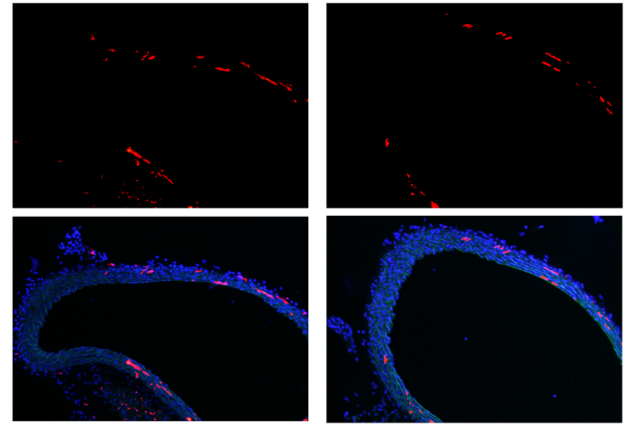

PDGF-BB Ab
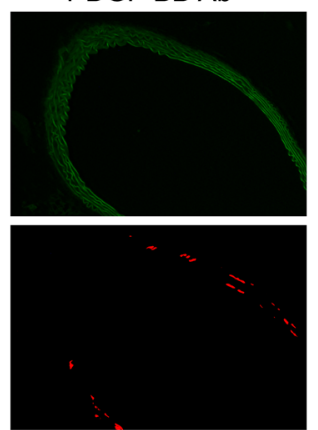

E

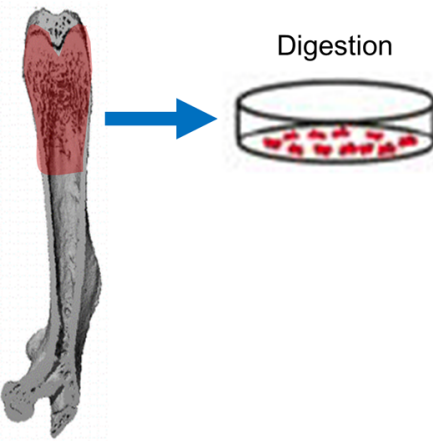

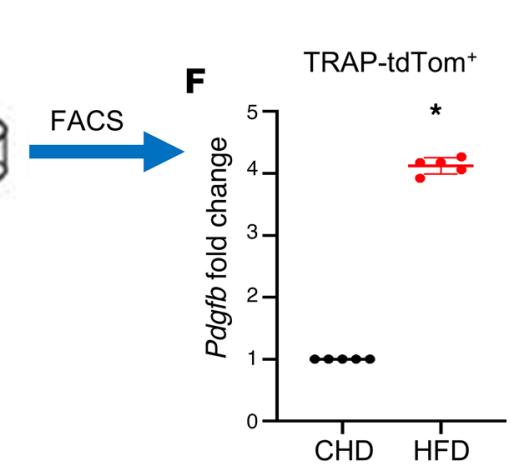
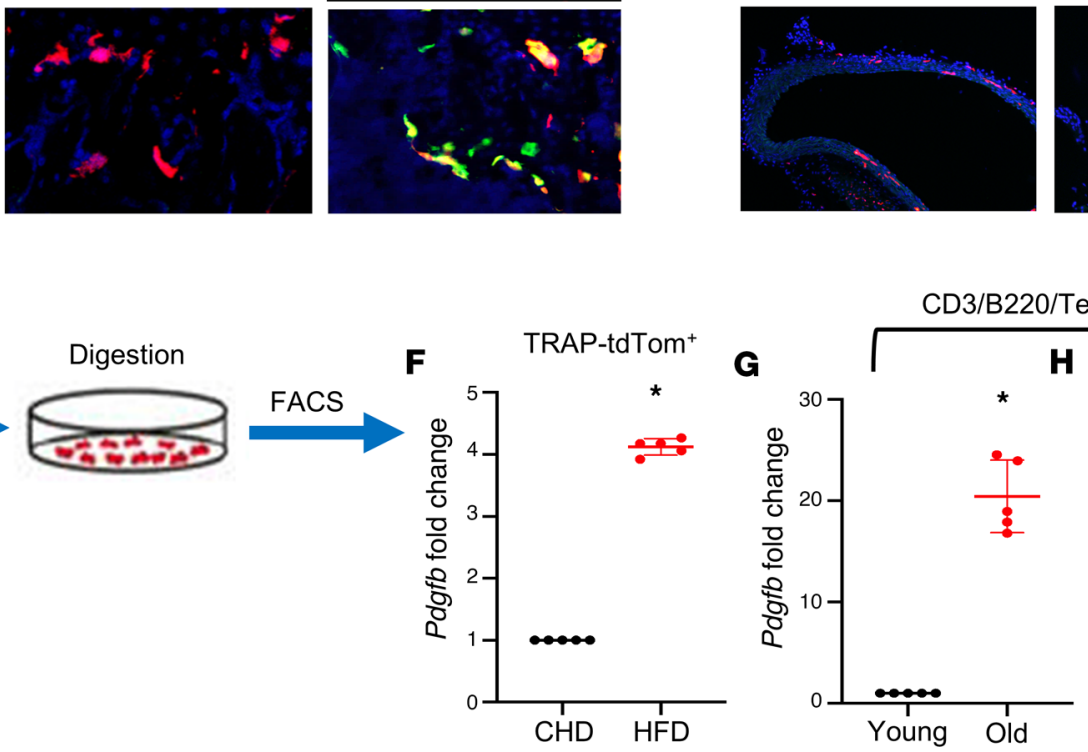

\section{H}

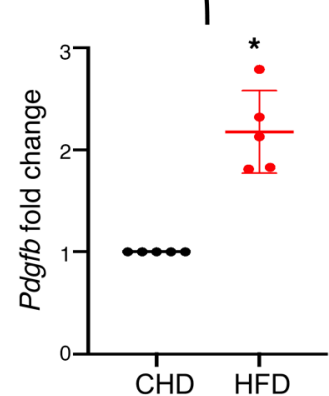

Figure 3. Bone/bone marrow preosteoclasts in aged mice and HFD mice are a main source of elevated circulating PDGF-BB. (A and B) Immunofluorescence staining of femoral bone tissue from 3 - and 20-month-old mice. Representative PDCF-BB staining image (A) and quantitative analysis of the number of PDGF-BB+ cells per tissue area (B). (C and $\mathbf{D})$ Frozen femoral bone (C) and aorta tissue sections (D) from TRAP/tdTom mice were subjected to immunofluorescence staining using specific PDGF-BB antibody. Fluorescence imaging of tdTom ${ }^{+}$cells (red), PDGF-BB ${ }^{+}$cells (green), and double positive cells (yellow) are shown. (E-H) Measurement of Pdgfb mRNA in bone/bone marrow preosteoclasts. Diagram showing the procedure for the isolation of bone/bone marrow cells from femoral bone using our previously described approach (E) (also see description in Methods). Cell suspension collected from TRAP/tdTom mice with CHD and HFD was subject to FACS to isolate tdTom+ cells. mRNA expression levels of Pdgfb were measured by qRT-PCR (F). Cell suspension collected from C57B/L6 mice was subject to FACS to isolate CD3/B220/T119-RANK ${ }^{+}$cells. The mRNA levels of $P d g f b$ in aged mice versus young mice $(\mathbf{G})$ and HFD mice versus CHD mice $(\mathbf{H})$ were measured by qRT-PCR. $n=5$. Data are mean \pm SD. ${ }^{*} P<0.001$, as determined by Student's $t$ tests. 

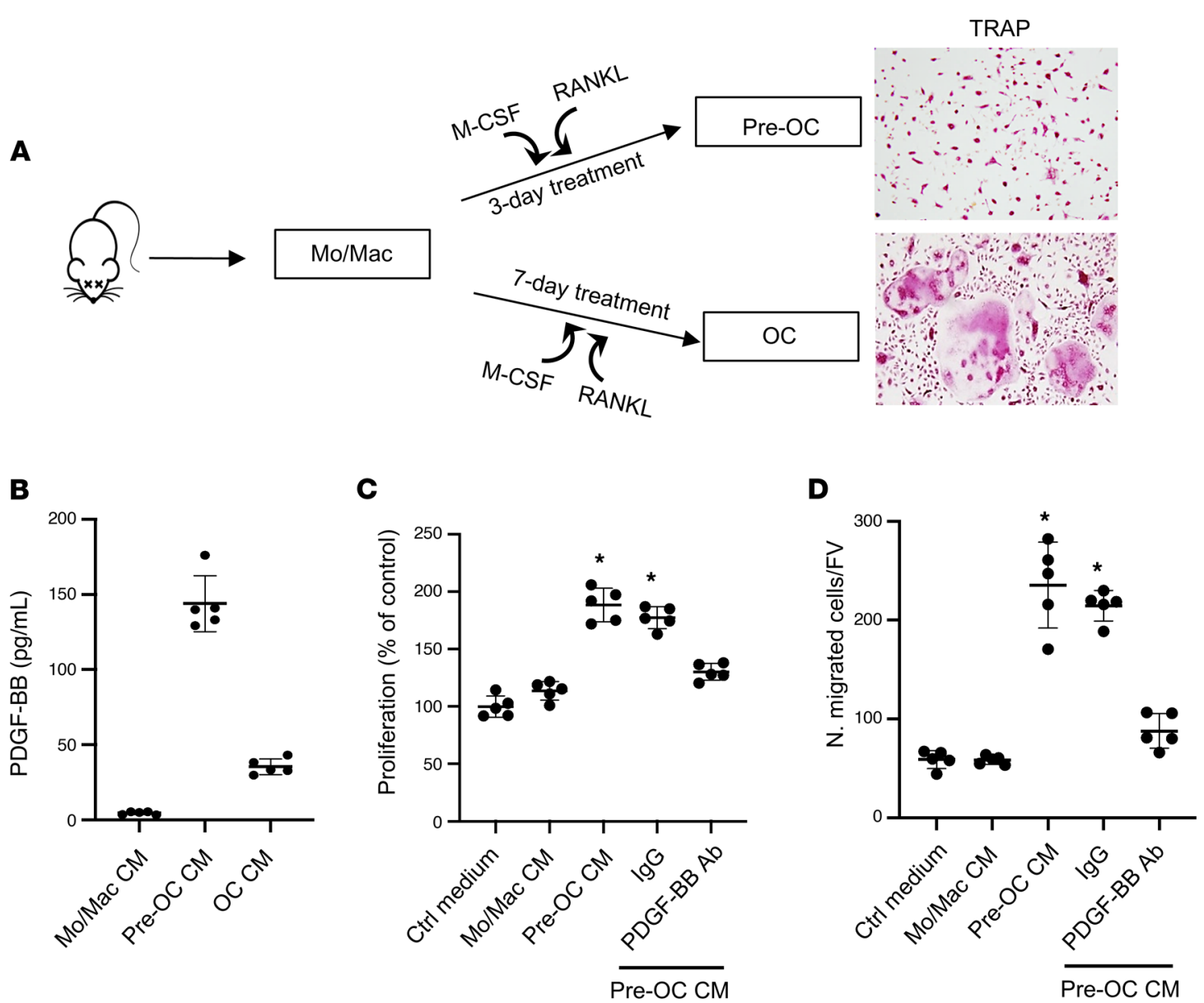

Figure 4. Preosteoclast-derived PDGF-BB stimulates VSMC proliferation and migration. (A) Schematic diagram showing the in vitro isolation of bone marrow Mo/Macs and the induction of osteoclast differentiation. (B) Conditioned medium (CM) was collected from Mo/Mac, preosteoclast, and osteoclast cultures, as described in Methods. PDGF-BB protein concentration in different CMs was measured using ELISA. (C) Rat VSMCs were incubated with CM from Mo/Mac, preosteoclasts, and osteoclasts for 48 hours. Cell proliferation was assessed using the MTT method. (D) Transwell assays for preosteoclast CM-induced migration of VSMCs. $n=5$. Data are mean \pm SD. ${ }^{*} P<0.05,1$-way ANOVA with Bonferroni post hoc test.

Conditional Pdgfb transgenic mice recapitulate low bone mass and an arterial stiffening phenotype. To determine whether increased production of PDGF-BB from preosteoclasts is sufficient to induce vascular stiffening, we generated conditional Pdgfb transgenic mice (Pdgfb ${ }^{\mathrm{cTG}}$ ), in which PDGF-BB is overexpressed in $\mathrm{TRAP}^{+}$cells by ligation of a 2.8-kb full-length human Pdgfb gene with a TRAP ${ }^{+}$cell-specific promoter, TRACP5 (29). No abnormal appearance or behavior was found in the Pdgfb ${ }^{\mathrm{cTG}}$ mice relative to their WT littermates. Intriguingly, bone marrow and serum PDGF-BB levels were more than 3-fold higher in the Pdgfb ${ }^{\mathrm{cTG}}$ mice compared with the age-matched WT mice (Figure 5A). We then assessed whether Pdgfb is specifically overexpressed in bone/ bone marrow preosteoclasts in the transgenic mice by measuring the mRNA expression in isolated bone/bone marrow CD3/B220/ Ter119-RANK ${ }^{+}$cells, which are primarily precursors of osteoclast lineage $(23,71,72)$. As we expected, quantitative RT-PCR analysis shows that $P d g f b$ expression was markedly higher in preosteoclasts from Pdgfb ${ }^{\text {TT }}$ mice relative to WT mice (Figure 5B). To assess whether circulating myeloid cells and vascular resident cells may also be the sources of elevated circulating PDGF-BB in the transgenic mice, periphery blood myeloid cells and aorta tissue were harvested from Pdgfb ${ }^{\mathrm{cTG}}$ mice and WT littermates. Although the expression of $P d g f b$ was also detected, the expression levels were not significantly elevated in both periphery blood myeloid cells (Figure 5C) and aorta tissue (Figure 5D) from Pdgfb ${ }^{\mathrm{cTG}}$ mice relative to WT mice. Consistently, the PDGF-BB protein expression was dramatically increased in bone/bone marrow cells as detected by immunofluorescence staining of femoral bone tissue sections (Figure 5E). Increased PDGF-BB expression was not found in aortic walls from Pdgfb ${ }^{\mathrm{TTG}}$ mice relative to WT mice (Figure 5F). Of note, $\mathrm{PDGF}-\mathrm{BB}^{+}$cells were not detected in any of the aortas where calcification was found in the Pdgfb ${ }^{\mathrm{cTG}}$ mice. Therefore, the elevated circulating PDGF-BB in transgenic mice is primarily produced by bone/bone marrow preosteoclasts rather than a local effect derived from blood vessels and blood myeloid cells.

We conducted a systemic bone phenotypic analyses of the transgenic mice at 6 months of age. MicroCT analyses of the distal femur in 6-month-old male Pdgfb ${ }^{\text {cTG }}$ mice revealed a low bone mass phenotype (Figure 6A) with reduced BV/TV (Figure 6B) and Tb.N (Figure 6D) and increased Tb.Sp (Figure 6E) relative to their WT littermates. Tb.Th was not changed in the Pdgfb ${ }^{\mathrm{cTG}}$ mice compared with the WT mice (Figure 6C). Therefore, young Pdg- 
A

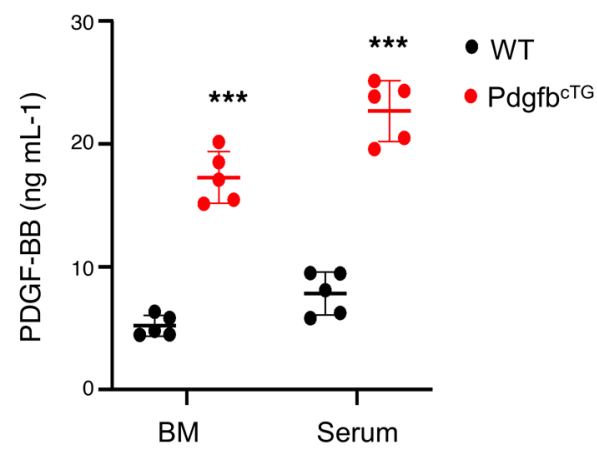

B

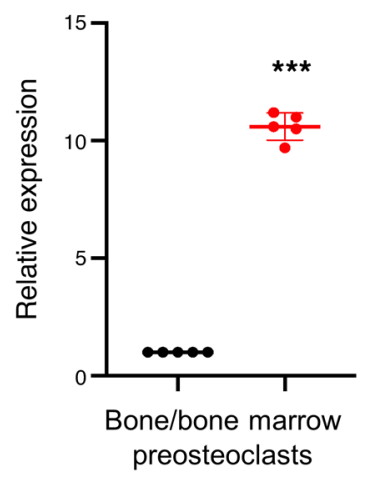

C

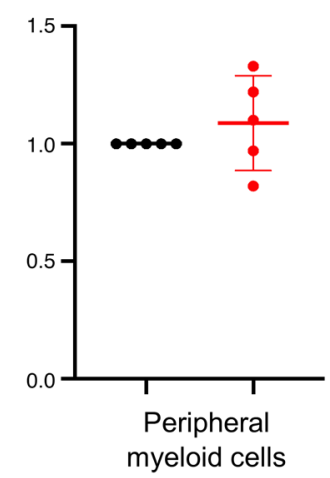

D

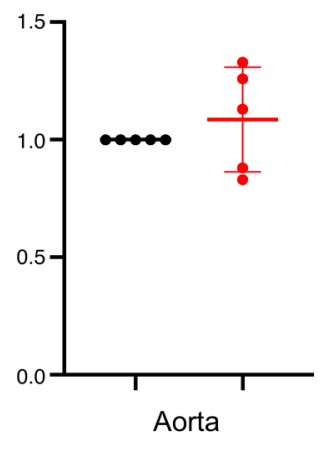

E

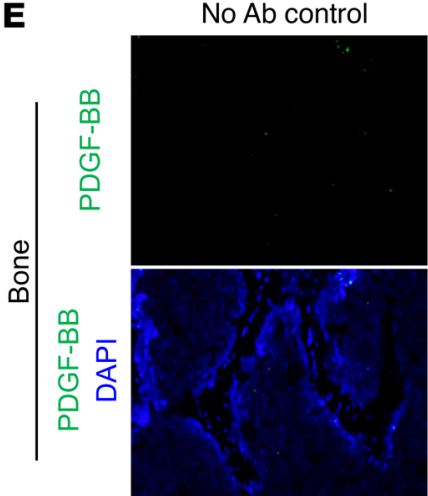

$\mathbf{F}$

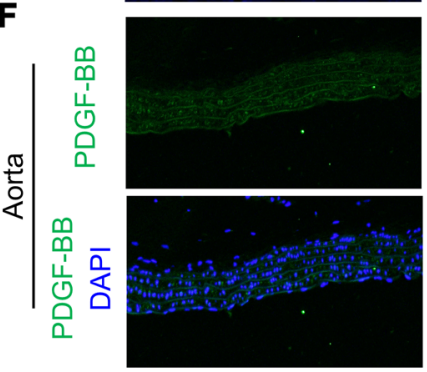

WT
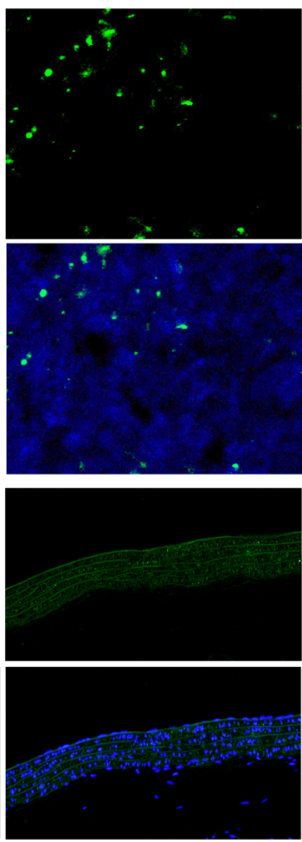

PdgfbcTG
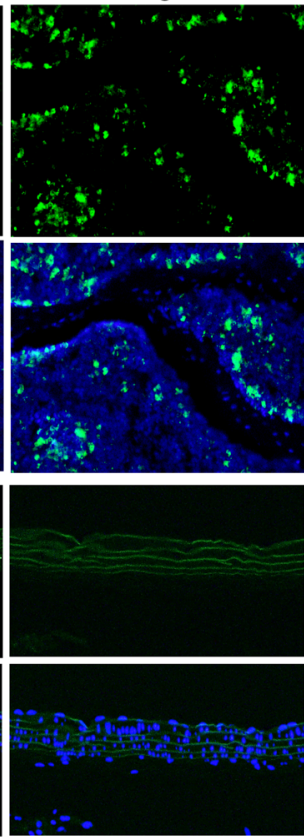

Figure 5. Conditional Pdgfb transgenic mice have increased PDGF-BB expression in bone/bone marrow and elevated serum PDGF-BB concentration. (A) ELISA measurements of bone marrow (BM) and serum PDGFBB concentrations in PdgfbcTC and WT littermates. (B-D) Bone/bone marrow CD3/B220/T119-RANK ${ }^{+}$cells (B), peripheral blood myeloid cells (C), and aorta tissue (D) were collected from 6-month-old Pdgfb ${ }^{\text {TTC }}$ mice and WT littermates as described in Methods. mRNA expression of $P d g f b$ was measured by qRT-PCR. (E and F) Representative PDCF-BB immunofluorescence staining of the femoral bone (E) and aorta (F) tissue sections from 6-month-old Pdgfb ${ }^{\text {TC }}$ mice and WT littermates. $n=5$. Data are mean \pm SD. ${ }^{* *} P<0.001$, as determined by Student's $t$ tests.
$\mathrm{fb}^{\mathrm{cTG}}$ mice mirrored aging-associated trabecular bone changes. Cortical thickness (Ct.Th) and bone area (B. Ar) were not different in the $\mathrm{Pdgfb}^{\mathrm{cTG}}$ mice compared with the WT mice (Figure 6, F-H). We also evaluated 9-month-old female mice and found that female Pdgfb ${ }^{\text {cTG }}$ mice had a similar low bone mass phenotype in trabecular compartment relative to their age- and sex-matched WT littermates (Figure 6, I-L). Therefore, overexpression of $P d g f b$ in the preosteoclasts results in decreased bone mass in trabecular but not in cortical bone compartments. Histomorphometry analysis shows that the number of bone surface osteocalcin $(\mathrm{OCN})^{+}$ osteoblasts were significantly reduced (Figure 6, M and N) but the number of bone surface $\mathrm{TRAP}^{+}$osteoclasts remained unchanged (Figure 6, O and $\mathrm{P}$ ) in the $\mathrm{Pdgfb}^{\mathrm{cTG}}$ mice relative to WT mice, suggesting that overexpression of $P d g f b$ in the preosteoclasts primarily impaired osteoblast bone formation.

We then measured PWV, the gold-standard index for aortic stiffness. Both young (3-4 months old) and old (>18 months old) $\mathrm{Pdgfb}^{\text {cTG }}$ mice had significantly higher PWV compared with their age-matched WT mice (Figure 7A). Moreover, PWV is also high- er in old Pdgfb ${ }^{\mathrm{cTG}}$ relative to young Pdgfb ${ }^{\mathrm{cTG}}$ mice, indicating an age-dependent progression of arterial stiffening in the conditional transgenic mice. As expected, PWV increased significantly with age in WT control mice. In the aged mice, a significant sex effect was noted as old female mice had significantly higher PWV than corresponding age-matched males (Figure 7E). Systolic, diastolic, and mean BPs were all significantly higher in young Pdgfb ${ }^{\mathrm{cTG}}$ mice than those in age-matched WT mice (Figure 7, B-D). However, differences in $\mathrm{BP}$ were not noted in the old Pdgfb ${ }^{\mathrm{cTG}}$ mice versus agematched WT mice. Aged female WT mice had significantly lower systolic, diastolic, and mean pressures than did aged male WTs. However, no sex differences were noted in the BP of Pdgfb ${ }^{\text {cTG }}$ old mice (Figure 7, F-H).

It has been reported that there is an age-dependent increase in the lumen diameter and wall thickness of the aorta $(73,74)$. Consistent with these previous reports, the thoracic aortic lumen diameter was greater in both aged (versus young) WT mice and aged (versus young) Pdgfb ${ }^{\text {cTG }}$ mice (Figure 8, A and B). Importantly, aged Pdgfb ${ }^{\text {cTG }}$ mice, relative to their age-matched WT littermates, 

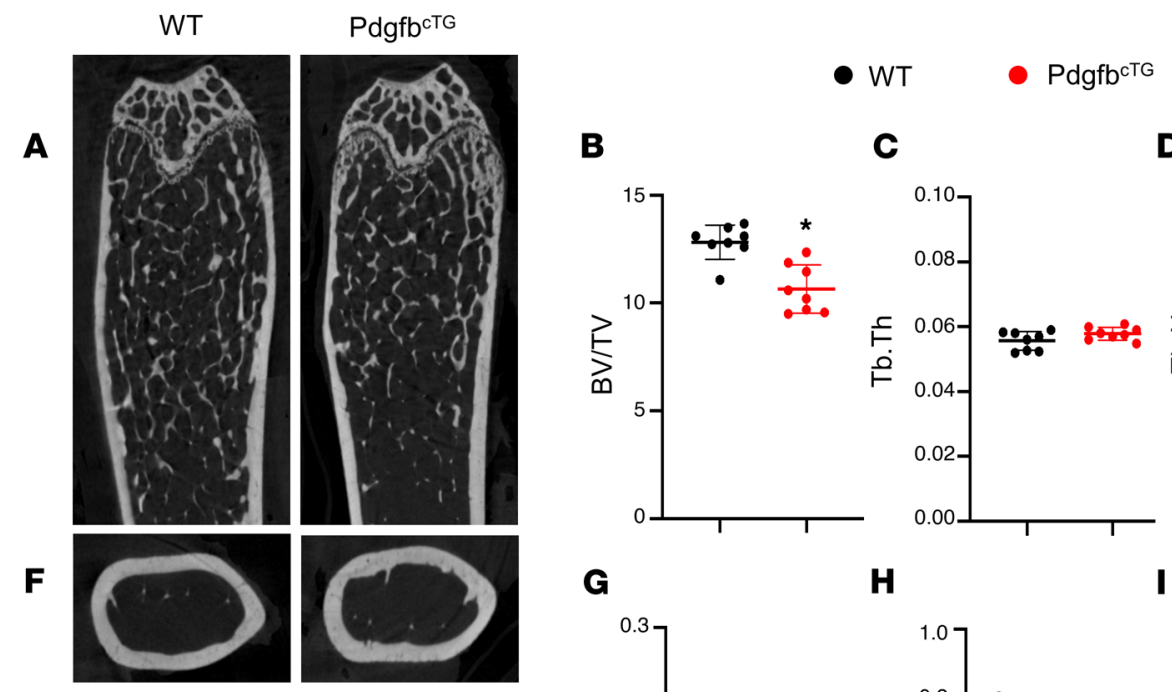

D
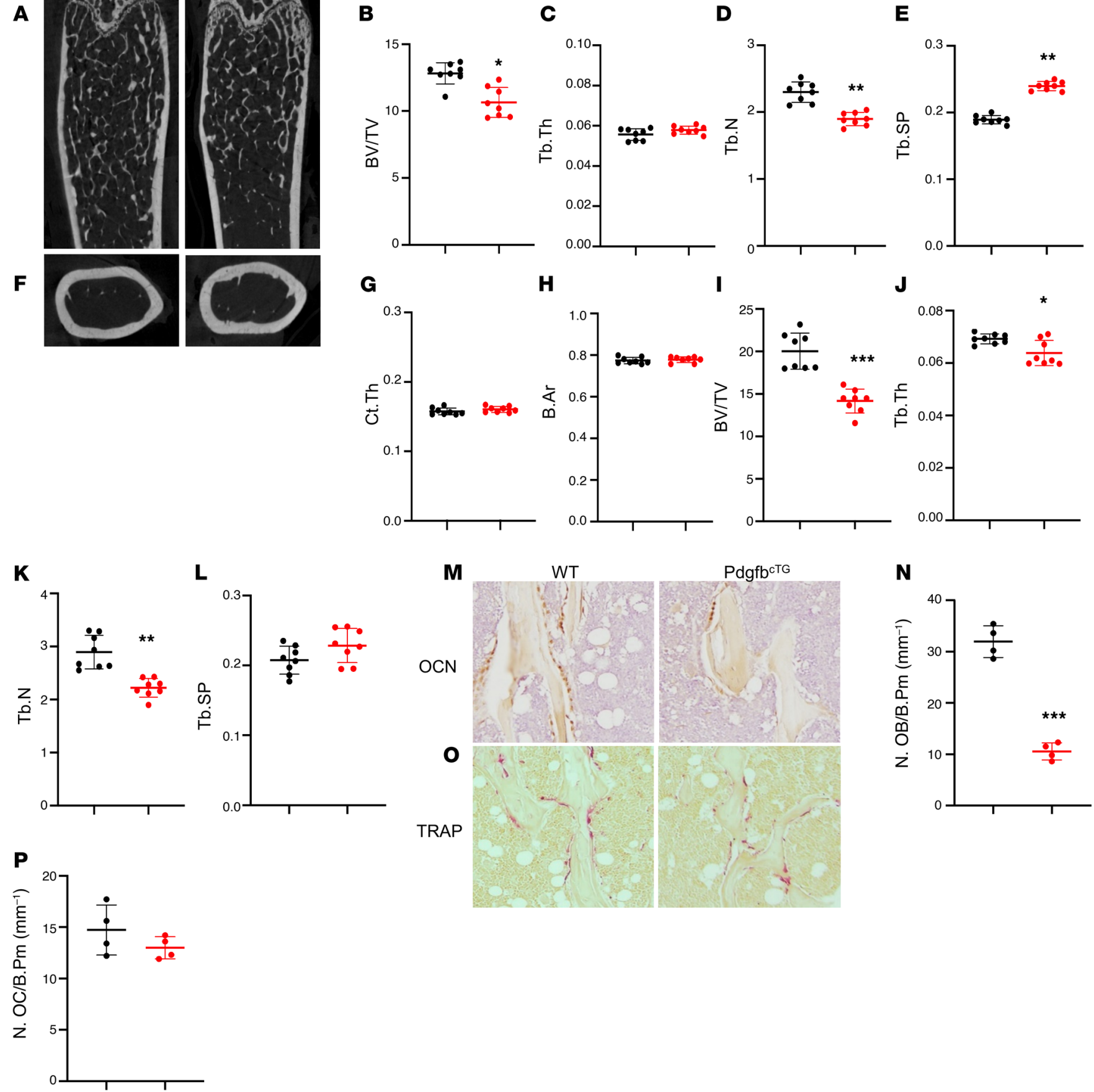

Figure 6. Conditional Pdgfb transgenic mice recapitulate an aging-associated bone phenotype. (A-E) Representative $\mu \mathrm{CT}$ images (A) and quantitative analyses (B-E) of the trabecular bone area of the distal femur from male 6-month-old PdgfbcTC mice and WT littermates. BV/TV (B), Tb.Th (C), Tb.N (D), and Tb.Sp (E). Representative $\mu C T$ images $(\mathbf{F})$ and quantitative analysis $(\mathbf{G}$ and $\mathbf{H})$ of the cross-sections of femoral mid-diaphysis of mice. Ct.Th, cortical bone thickness; B. Ar, bone area. (I-L) Quantitative $\mu$ CT analyses of the trabecular bone area of the distal femur from female 9-monthold Pdgfb cTC mice and WT littermates. BV/TV (I), Tb.Th (J), Tb.N (K), and Tb.Sp (L). (M and $\mathbf{N})$ Representative immunohistochemical staining (M) and quantitative analysis of osteocalcin $(\mathrm{OCN})(\mathbf{N})$ in femur sections. ( $\mathbf{O}$ and $\mathbf{P})$ Representative TRAP staining $(\mathbf{O})$ and quantitative analysis of TRAP+ ${ }^{+}$cells in femur sections (P). N.OB/B.Pm, number of osteocalcin ${ }^{+}$osteoblasts per bone perimeter; N.OC/B.Pm, number of TRAP ${ }^{+}$osteoclasts per bone perimeter. ${ }^{*} P<0.05,{ }^{*} P<0.01,{ }^{* *} P<0.001$ as determined by Student's $t$ tests. 
A

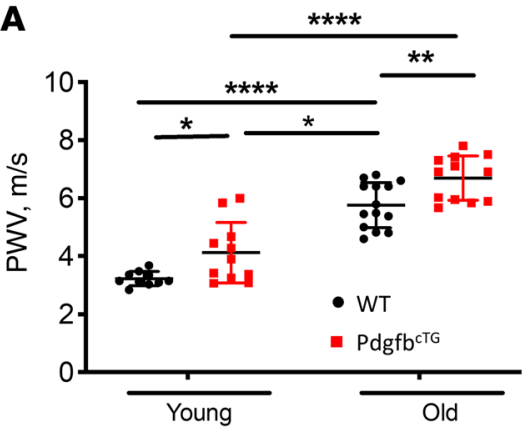

B
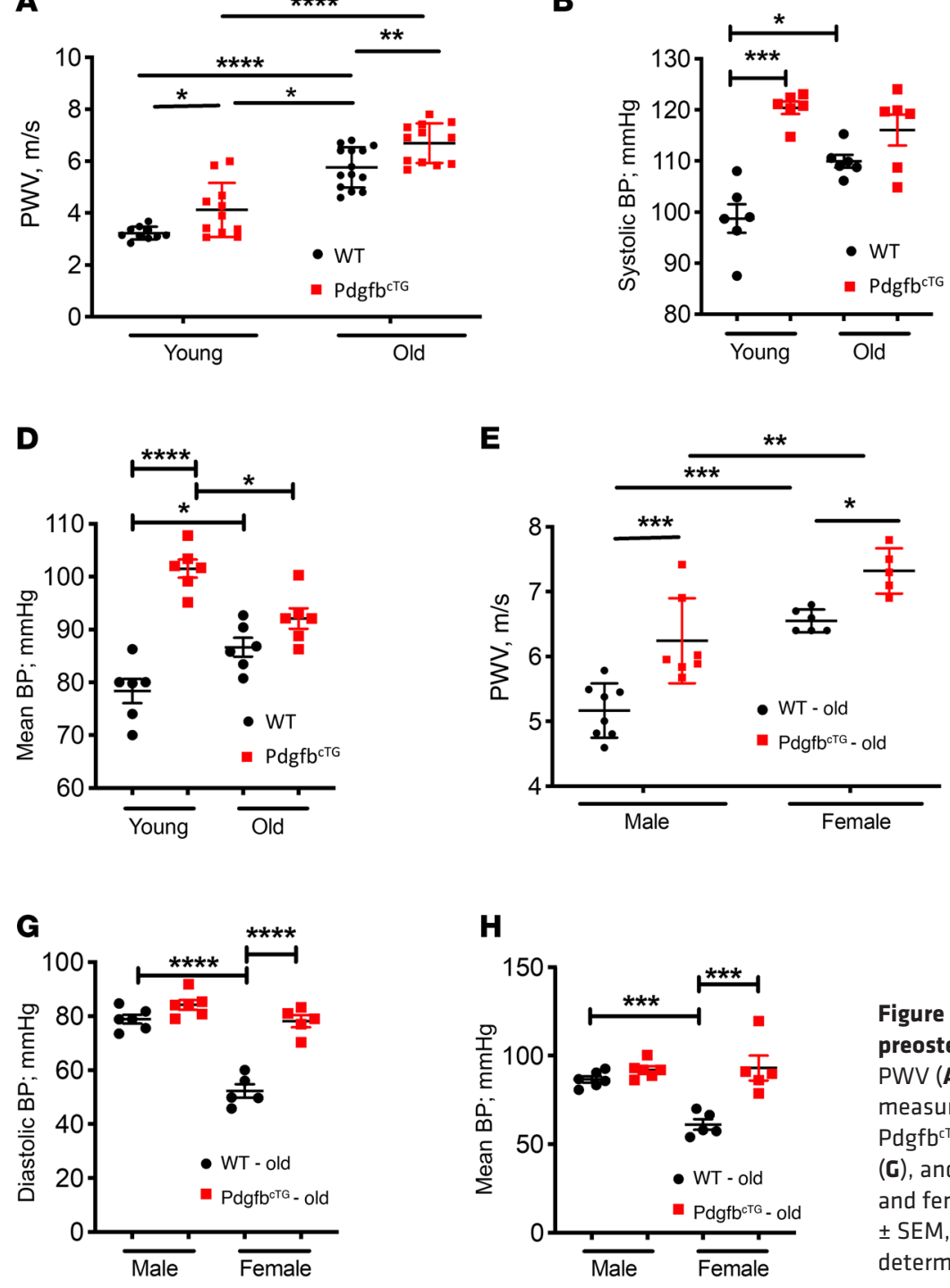

E

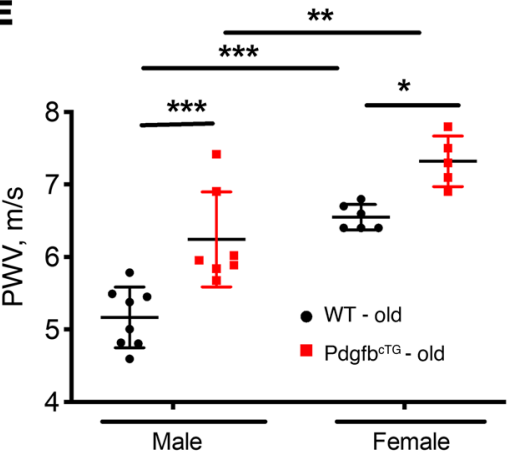

H

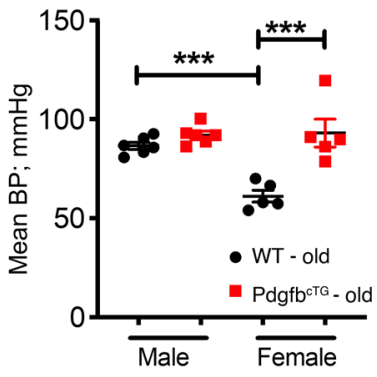

C

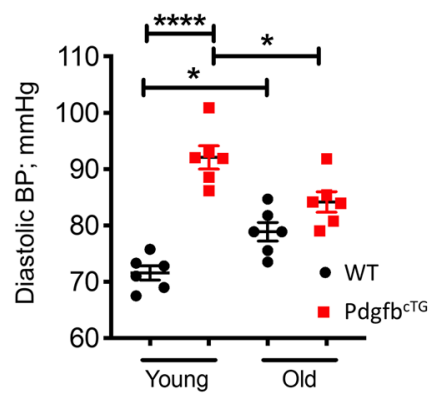

$\mathbf{F}$

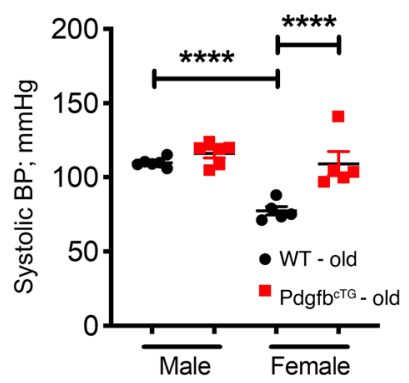

Figure 7. Conditional transgenic mice expressing PDGF-BB in preosteoclasts recapitulate aging-associated artery phenotype. PWV (A), systolic BP (B), diastolic BP (C), and mean BP (D) were measured in young (3- to 4-month old) and old ( $>18$ months old) Pdgfbctc and WT littermates. PWV (E), systolic BP (F), diastolic BP (G), and mean BP (H) were measured in old ( $>18$ months old) male and female PdgfbcTC and WT littermates. $n=5$ to 14. Data are mean \pm SEM, ${ }^{*} P<0.05,{ }^{* *} P<0.01,{ }^{* * *} P<0.001,{ }^{* * *} P<0.0001$ as determined by 1-way ANOVA with Bonferroni post hoc test. had increased aortic lumen diameter, indicating an exacerbated age-related morphological change of the aorta when Pdgfb is overexpressed. Aortic wall was significantly thicker in aged WT and Pdgfb ${ }^{\text {TTG }}$ mice than in young WT and Pdgfb ${ }^{\text {cTG }}$ mice, respectively (Figure 8, A and C). The aortas from aged WT and Pdgfb ${ }^{\text {сTG }}$ mice, relative to young mice, showed smooth muscle cell nuclei loss (Figure 8D), a characteristic of vascular aging. Moreover, the VSMC nuclei loss is more in the WT mice than in the Pdgfb ${ }^{\mathrm{cTG}}$ mice, indicating that the old Pdgfb ${ }^{\mathrm{cTG}}$ mice may have increased PDGFB/ PDGFR $\beta$ signaling in the arterial tissue. Lamellar thickness and intralamellar distance both increased significantly with age in the WT and $\mathrm{Pdgfb}^{\text {cTG }}$ mice, indicating a significant accumulation of matrix in the vascular wall (Figure 8, E and F). Vascular calcification is a key link between osteoporosis and CVD. We then assessed whether the transgenic mice have vascular calcification by performing von Kossa staining of aorta tissue sections. Positive signal was found in 1 of 4 old mice ( 24 months old) but in 0 of 6 young mice (4 months old). Importantly, positive signal was found in 2 of $8 \mathrm{Pdgfb}^{\mathrm{cTG}}$ mice at 6 months of age, whereas none of the 7 aortas from the littermates (WT) showed positive signaling (Figure $8 \mathrm{G}$ ).
In addition, we examined the activation of an osteogenic trans-differentiation program in the aortas of the $\mathrm{Pdgfb}^{\mathrm{cTG}}$ mice. Both osteoblast differentiation markers RUNX2 (Figure 8H) and ALP (Figure 8I) were upregulated at mRNA level in the aortas of Pdgfb ${ }^{\text {cTG }}$ mice compared with WT mice, indicating that vascular calcification may contribute to arterial stiffening induced by preosteoclast-secreted PDGF-BB. We also examined whether the expression of PDGFR $\beta$, the receptor of PDGF-BB, is changed in aorta tissues of old (versus young) and transgenic mice (versus WT mice). Markedly increased expression of PDGFR $\beta$ in smooth muscle cells of the aorta wall was found in both aged mice and $\mathrm{Pdgfb}^{\mathrm{cTG}}$ mice, as compared with young mice and WT mice, respectively (Figure 8J).

We then measured the passive stiffness of the vessels. Tensile testing showed greater stiffness of the descending aorta in both young and aged Pdgfb ${ }^{\text {сTG }}$ mice compared with their age-matched WT littermates, with the difference in young mice being of higher magnitude (Figure 9, A and B). While there was a greater vessel stiffness in the aged WT mice relative to young WT mice, this age-dependent difference was not significant in the Pdgfb ${ }^{\text {cTG }}$ mice (Figure 9, C and D). The significantly higher passive stiffness of the 
A

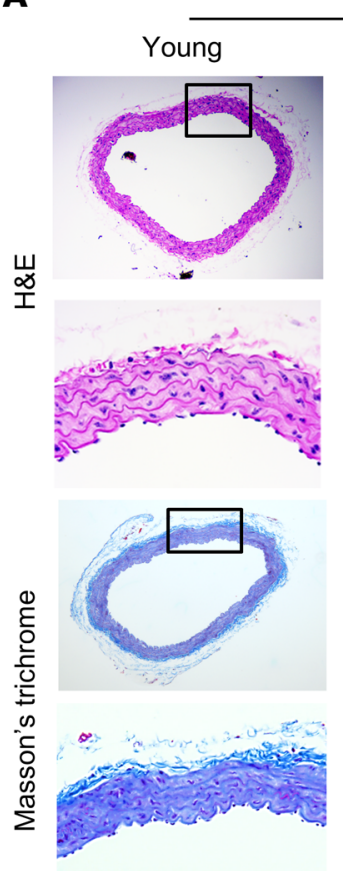

D

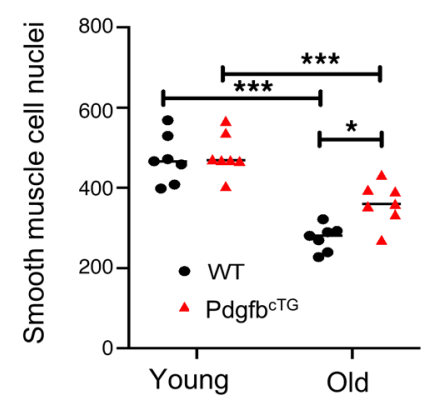

G
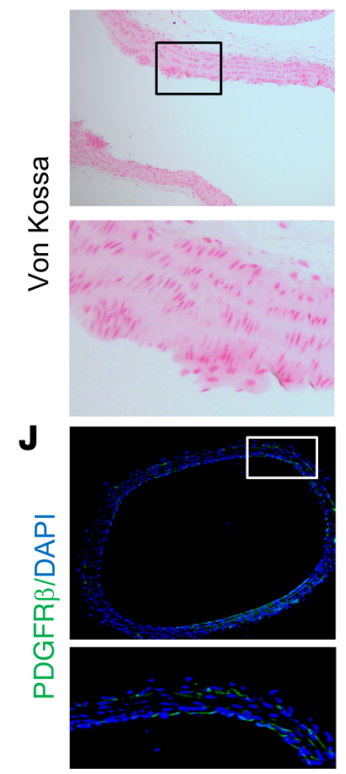

WT
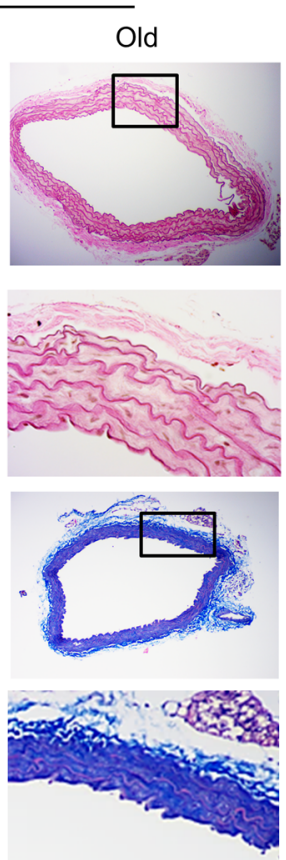

E
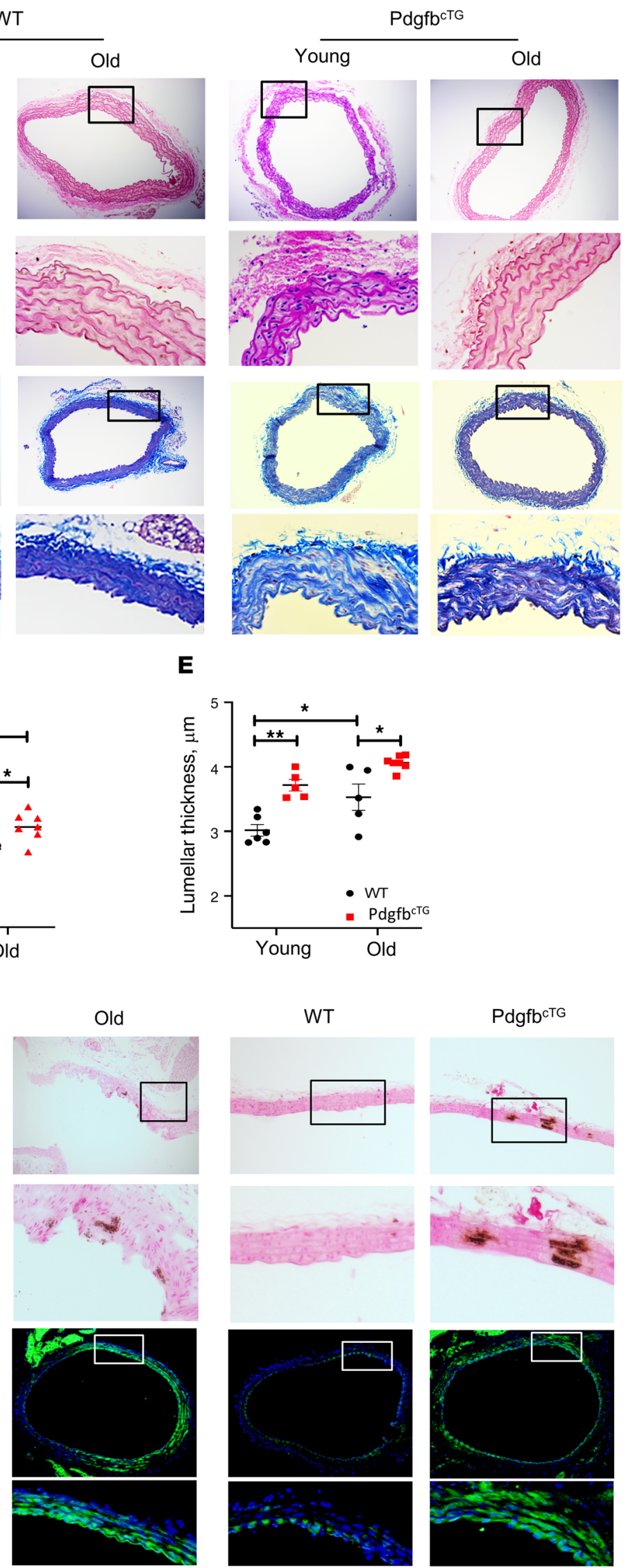

WT
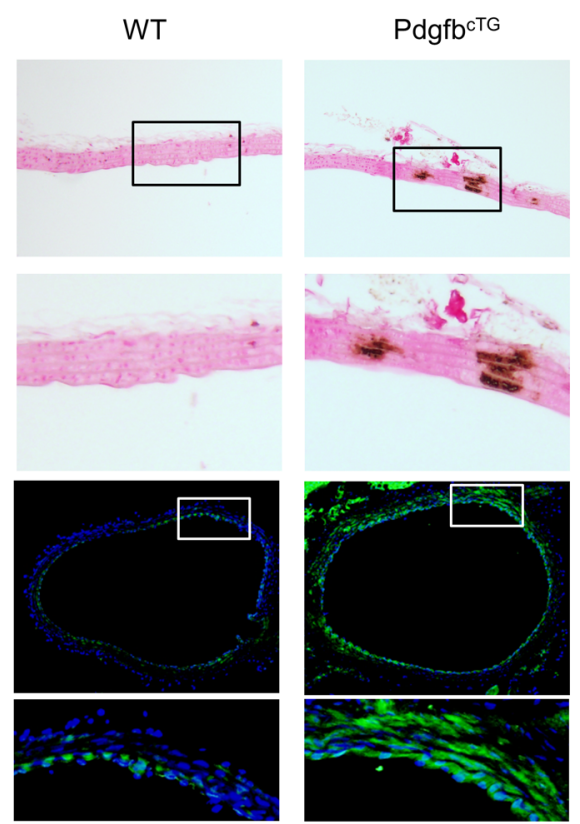

B
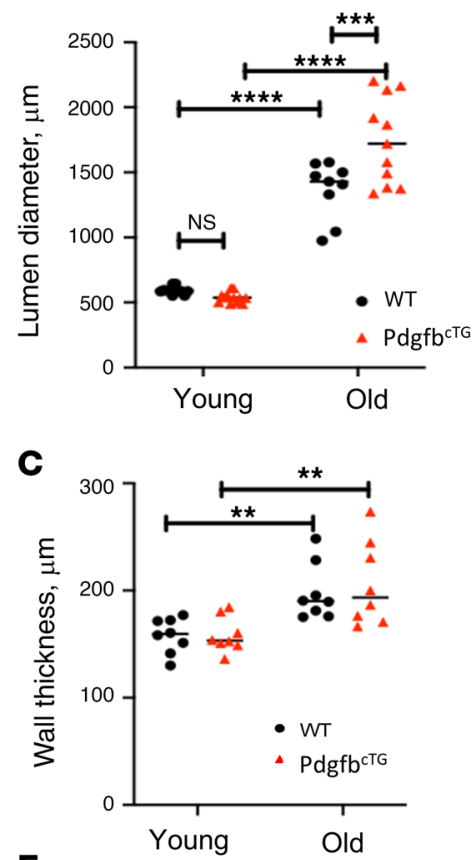

F

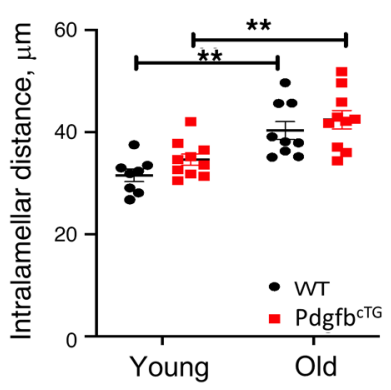

H
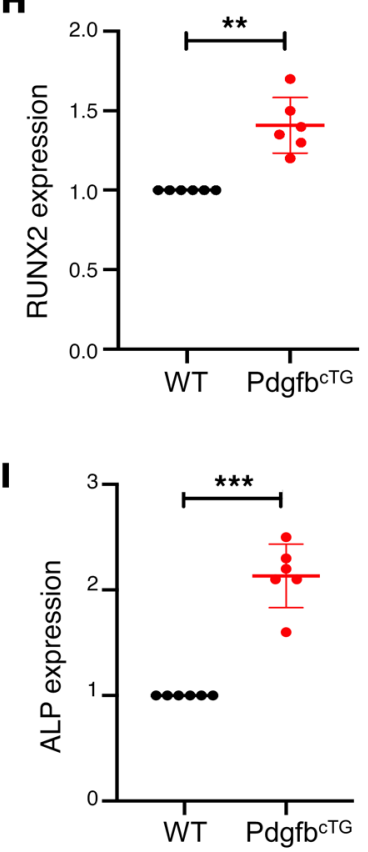
Figure 8. Conditional Pdgfb transgenic mice develop pathological aortic morphology and vascular calcification. (A) Representative histological staining analysis (10x; inset $40 x$ ) showing H\&E and Masson's trichrome staining of aorta from 4- and 18-month-old PdgfbcTC and WT littermates. (B) Lumen diameter, (C) vessel wall thickness, and (D) smooth muscle cell nuclei, Lamellar thickness (E), and intralamellar distance $(\mathbf{F})$ in aortas were calculated. $n=5$ to $11 .{ }^{*} P<0.05$, ${ }^{* *} P<0.01$, ${ }^{* *} P<0.001$, and ${ }^{* * * *} P<0.0001$ as determined by 1 -way ANOVA with Bonferroni post hoc test. (G) Representative micrographs of von Kossa stained sections of the thoracic aorta from young (4-month-old) and old (20-month-old) mice (left panels) and from 6-month-old Pdgfb ${ }^{\text {TTC }}$ and WT littermates (right panels). (H and I) Aorta tissues were harvested from 6-month-old Pdgfb ${ }^{\text {cTC }}$ and WT littermates. mRNA expressions of RUNX2 (H) and alkaline phosphatase (ALP) (I) were measured by qRTPCR. $n=6$. Data are mean $\pm \mathrm{SD},{ }^{*} P<0.01,{ }^{* *} P<0.001$ as determined by Student's $t$ tests. (J) Immunofluorescence staining of aortic tissue sections with antibody against PDCFR $\beta$ from young (4-month-old) and old (20-month-old) mice (left panels) and from 6-month-old Pdgfb ${ }^{\text {cTC }}$ and WT littermates (right panels).

Pdgfb $^{\mathrm{cTG}}$ mouse aorta determined by tensile testing suggests that the increase in PWV noted in these mice is not solely due to the effect of higher BP, and there is substantial arterial stiffening as well.

We also tested the contraction and relaxation responses of the aorta. We did not detect differences in the contraction response to phenylephrine (Figure 9E) and endothelial relaxation response to acetylcholine (Figure 9G) of the vessels from the young Pdgfb ${ }^{\text {cTC }}$ mice compared with those from the young WT mice. However, phenylephrine-induced contraction was higher in the vessels from the aged Pdgfb ${ }^{\text {cT }}$ mice relative to the age-matched WT mice (Figure $9, \mathrm{~F}$ and $\mathrm{H}$ ), suggesting that $P d g f b$ overexpression sensitizes the vessels to agonist-induced contraction in aging, as has been previously shown (75). This suggests the possibility of augmented tone of the vascular smooth muscle cells with increased circulating PDGF-BB. Moreover, acetylcholine-mediated relaxation of preconstricted vessels was notably lower in the aorta from aged Pdgfb $^{\text {cTG }}$ mice when compared with age-matched WT controls when Cox pathways were inhibited using indomethacin. No differences were noted in the endothelial-independent sodium nitroprusside-induced (SNP-induced) relaxation in both young and aged Pdgfb ${ }^{\text {TTG }}$ mice relative to age-matched WT mice (Figure 9, I and J). This suggests that Pdgfb either induces a larger deficit in age-associated endothelial dysfunction, or that the larger preconstriction caused by phenylephrine is not fully countered by the endothelial-mediated relaxation response. Together, these findings suggest that Pdgfb overexpression in preosteoclasts causes endothelial dysfunction, VSMC dysregulation, and vascular stiffening during aging.

Conditional Pdgfb knockout mice are protected from HFD-induced bone loss and arterial stiffening. We tested whether increased circulating PDGF-BB is required for HFD-induced bone loss and arterial stiffening by generating conditional Pdgfb knockout mice (Pdg$\mathrm{fb}-\mathrm{cKO}$ ), in which $P d g f b$ is deleted selectively in the $\mathrm{TRAP}^{+}$cells by crossing Pdgf $b^{f / f l}$ mice with Trap-Cre mice (29). We detected a dramatically lower mRNA level of Pdgfb in CD3/B220/Ter119-RANK ${ }^{+}$ preosteoclasts isolated from the bone/bone marrow of Pdgfb-cKO mice compared with $P d g f b^{f / f l}$ littermates (WT; Figure 10A), validating the efficiency of $P d g f b$ deletion in preosteoclasts in the knockout mice. Importantly, the serum PDGF-BB level was higher in WT mice fed a HFD compared with WT mice fed a CHD, but this elevation was not detected in Pdgfb-cKO mice after HFD challenge (Figure 10B). Of note, serum PDGF-BB concentration in HFD-challenged Pdgfb-cKO mice was reduced to a similar level as in the WT mice without HFD challenge. The results further validated that bone/ bone marrow preosteoclasts are a main source of elevated PDGF$\mathrm{BB}$ in blood circulation in mice during aging or under HFD.

We conducted an analysis of bone phenotype in Pdgfb-cKO mice (Figure 10C). MicroCT analyses shows that trabecular BV/ TV, Tb.N, and Tb.Th were all lower, and Tb.Sp was bigger in the distal femur of Pdgfb-cKO mice relative to their Pdgf $b^{f / f l}$ littermates (WT; Figure 10, D-G). The results are consistent with our previous work showing that healthy, unchallenged Pdgfb-cKO mice exhibited a low-bone-mass phenotype (28). We then evaluated whether deletion of $P d g f b$ from preosteoclasts affects bone phenotype in HFD-challenged mice, in which PDGF-BB concentrations in both bone marrow and serum were aberrantly elevated compared with mice fed normal CHD. Whereas HFD induced reduction in BV/ TV and Tb.N and increase in Tb.Sp in WT mice, the changes of these parameters induced by HFD were, at least partially, rectified by Pdgfb deletion (Figure 10, D-G). HFD induced an increase in cortical bone area (Ct. Ar) without changing cortical bone thickness (Ct.Th) in both WT and Pdgfb-cKO mice (Figure 10, $\mathrm{H}^{-} \mathrm{J}$ ). Therefore, preosteoclast-derived PDGF-BB plays a paradoxical role specifically in trabecular bone regulation. While PDGF-BB is required for the maintenance of bone homeostasis under normal physiological conditions, excessive production of PDGF-BB from preosteoclasts leads to trabecular bone loss in pathological conditions (e.g., aging and metabolic dysregulation).

We next determined whether targeting PDGF-BB secretion by preosteoclasts can prevent deterioration of vascular mechanics and function. To this end, we used a HFD challenge to accelerate vascular stiffening and deterioration of bone as a rapid alternative to natural aging, which takes at least 18 months in the mouse model. The HFD challenge is shown to cause an increase in PWV prior to the onset of systolic hypertension, as is the case in aging (35). Here, we measured PWV in Pdgfb-cKO mice fed a HFD for different time periods. $P d g f b^{f / f l}(\mathrm{WT})$ mice had a slight increase in PWV after 8 weeks and a significant increase in PWV after 12 to 14 weeks of HFD feeding. Importantly, Pdgfb-cKO mice were partially protected from the diet-induced elevation in PWV noted in the WT mice (Figure 11A). The difference in PWV levels at baseline between Pdgfb-cKO mice and WT mice was not significant. HFD-induced elevation of systolic, diastolic, and mean BPs in the WT mice was also not detected in the Pdgfb-cKO mice (Figure 11, B-D). Increased stiffness of both the matrix and VSMC dysfunction are known to occur in response to HFD, contributing to vascular stiffening in vivo. Therefore, we next examined the mechanical and functional properties of the aorta at the end of 14 weeks of HFD. Tensile testing of the descending aorta showed a significantly more compliant vessel in HFD Pdgfb-cKO mice compared with HFD WT mice (Figure $11 \mathrm{E})$. Vascular contractility studies showed an exaggerated contractility response to increasing concentrations of phenylephrine in WT mice compared with Pdgfb-cKO mice (Figure 11F). The endothelial-dependent relaxation to acetylcholine after preconstriction with phenylephrine was higher in Pdgfb-cKO mice than 
A

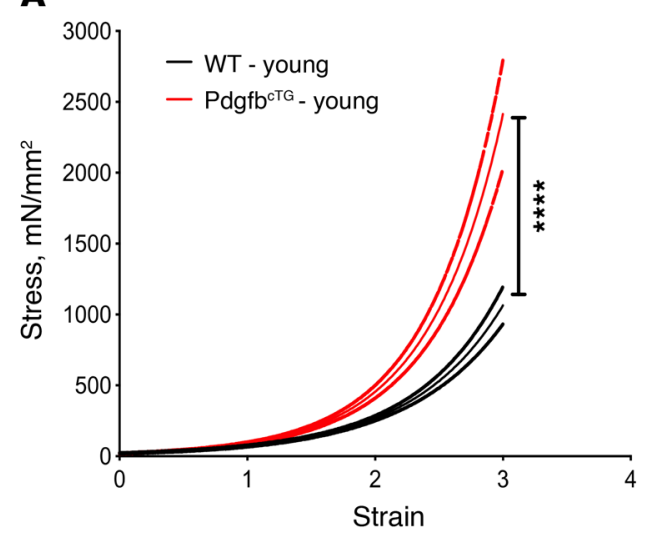

C

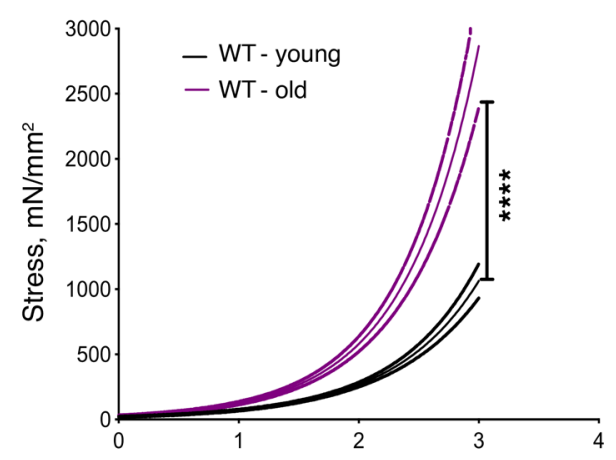

E

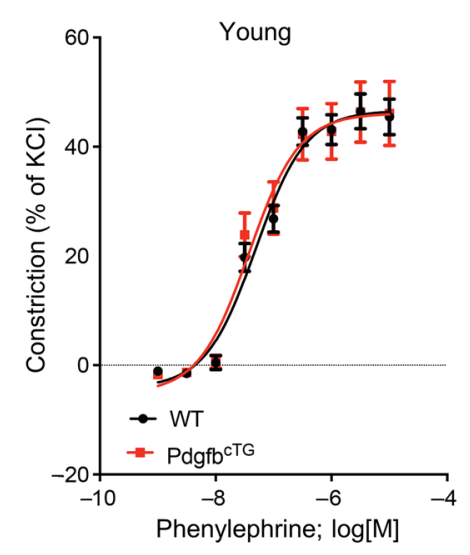

H

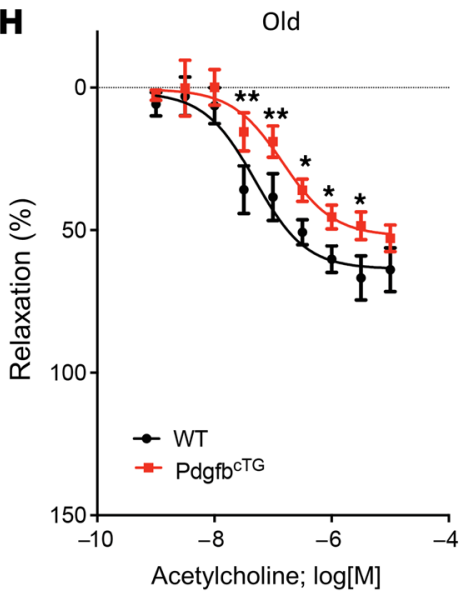

B

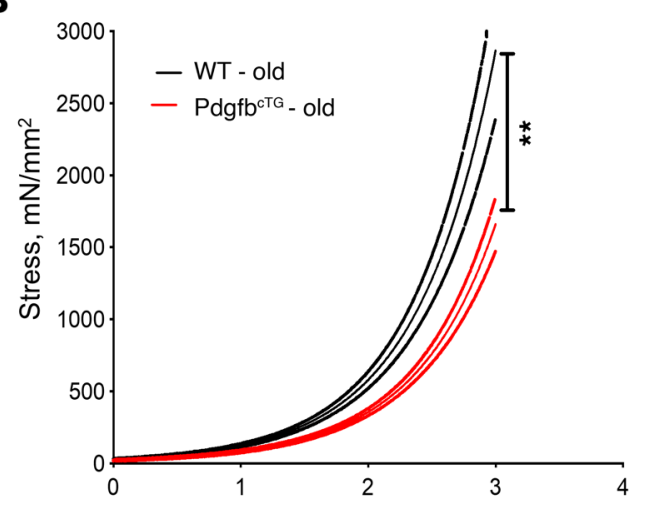

D

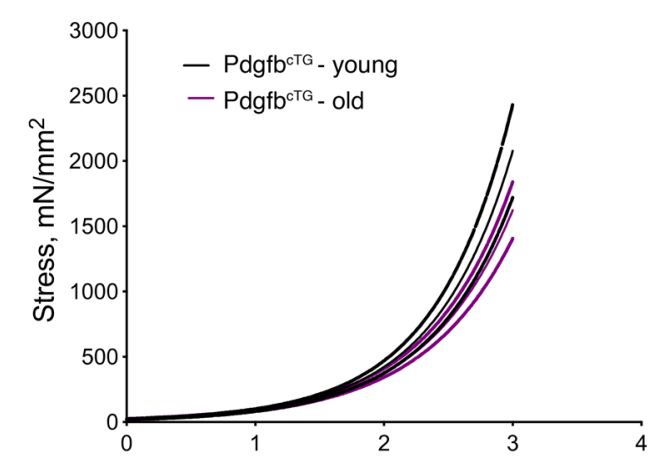

G
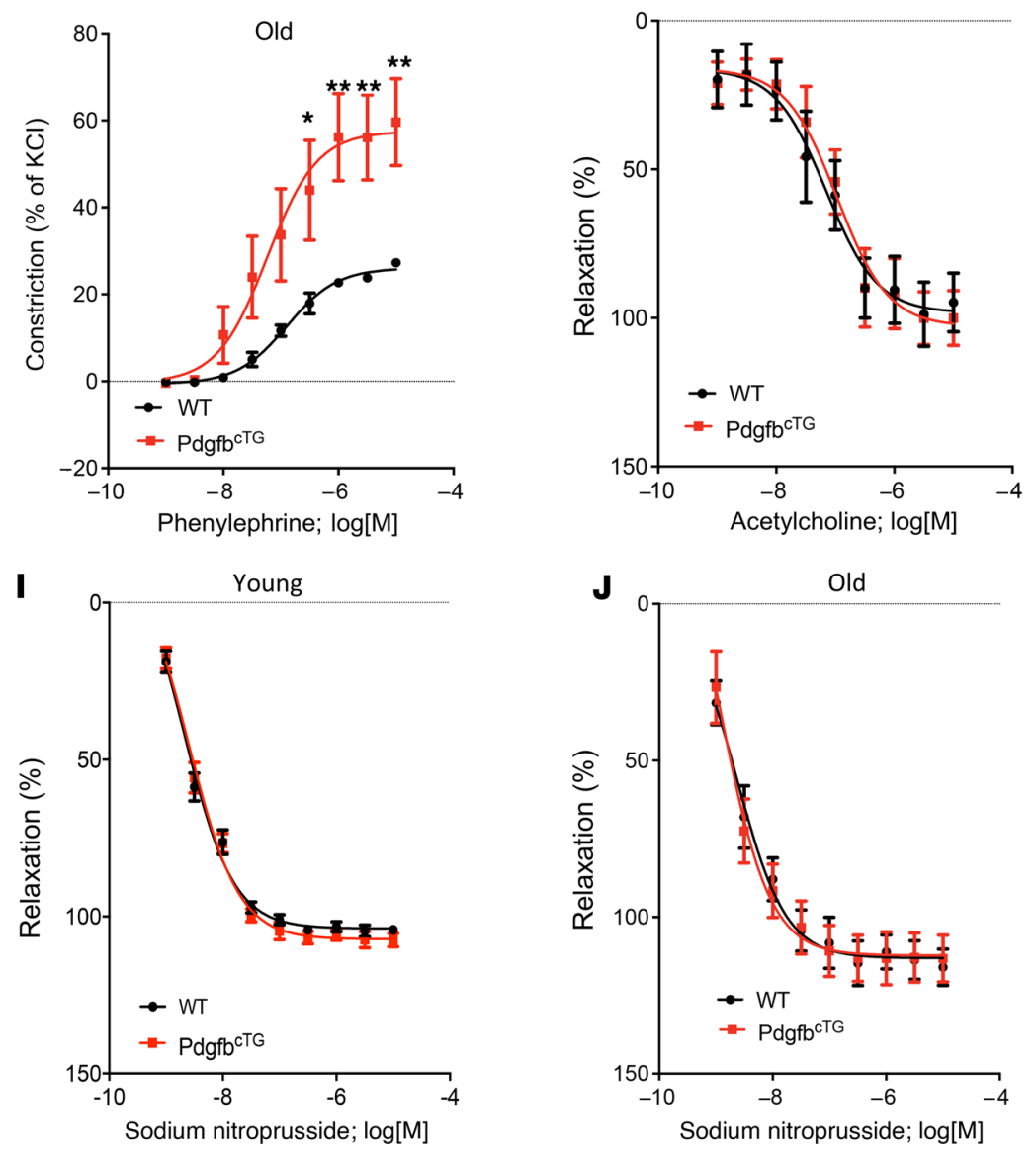
Figure 9. Aortic plasticity and vasoreactivity are impaired in conditional Pdgfb transgenic mice. (A-D) Tensile testing of aortic rings was measured in 4- and 18-month-old Pdgfbctc and WT littermates. $n=10$ rings. Data are mean $\pm \mathrm{SEM},{ }^{* * *} P<0.0001$ versus WT mice, as determined by 1 -way ANOVA with Bonferroni post hoc analysis. Aortic constriction in response to increasing doses of phenylephrine was measured in the young $(\mathbf{E})$ and aged (F) Pdgfb ${ }^{\text {cTC }}$ mice vs. WT littermates. $n=8$ aortic rings. Data are shown as mean $\pm \mathrm{SEM},{ }^{*} P<0.05$, ${ }^{*} P<0.01$, as determined by one-way ANOVA with Bonferroni post hoc test. Endothelium-dependent aortic relaxation in response to increasing doses of acetylcholine was measured in young $(\mathbf{G})$ and aged $(\mathbf{H})$ Pdgfb ${ }^{\text {cTC }}$ mice versus WT littermates. $n=8$ aortic rings. Data are mean $\pm \mathrm{SEM},{ }^{*} P<0.05,{ }^{* *} P<0.01$, as determined by 1 -way ANOVA with Bonferroni post hoc test. Endothelium-independent vasorelaxation in response to sodium nitroprusside was measured in young (I) and aged (J) PdgfbcT mice versus WT littermates. $n=8$ aortic rings. Data are mean \pm SEM.

in WT mice (Figure 11G). The endothelial-independent relaxation of the vessels to SNP was similar between the 2 groups (Figure 11H), suggesting that the blunted acetylcholine response of WT mice is caused by a greater decline in the endothelial function of WT mice in response to a HFD than in the Pdgfb-cKO mice. Together, these findings suggest that PDGF-BB promotes diet-induced vascular stiffening by mediating changes in cellular function, including augmented vascular contractility in conjunction with endothelial dysfunction in the HFD WT mice.

\section{Discussion}

The regulatory mechanisms of the vascular system through bone-derived cues during aging are poorly understood. Here we showed that with advancing age or under metabolic stress, mononuclear preosteoclasts in bone/bone marrow, as a main source of excessive circulating PDGF-BB, contribute to arterial stiffening (Figure 12). Our study provides new insight into the cellular and molecular mechanisms underlying the bone/vascular axis. Despite the well-recognized role of PDGF-BB in aging-associated arterial stiffness and atherosclerosis development, we are aware of no studies of the role of PDGF-BB as a systemic proaging factor. We detected elevated bone marrow and serum PDGF-BB concentration in aged mice, rats, and human subjects relative to their young counterparts. More importantly, the data from our conditional transgenic and knockout mouse models suggest that aberrantly elevated PDGF-BB secreted by preosteoclasts is a driving force for the pathological changes of both the skeletal and vascular systems (i.e., bone loss and arterial stiffening) in response to aging and HFD challenge.

Multinuclear osteoclasts have been considered an orchestrator, with more functions beyond bone resorption. Osteoclasts secrete clastokines that regulate the activity of neighboring cells within the bone/bone marrow microenvironment (20). It was reported that PDGF-BB in bone/bone marrow microenvironment is primarily produced by osteoclast precursors but not by uncommitted Mo/Macs and multinuclear osteoclasts in healthy, young mice (28). The present study agrees with this finding and further demonstrates that mononuclear $\mathrm{TRAP}^{+}$preosteoclasts secrete much more PDGF-BB in aged mice and HFD-challenged mice relative to young mice and CHD mice, respectively. The mechanisms by which preosteoclasts secrete a high amount of PDGF-BB during aging or under metabolic stress remain unclear. Preosteoclasts may develop a unique secretory phenotype during aging and are likely a primary cell type producing clastokines to regulate other cell types or tissues. Further analysis of the other factors released from preosteoclasts in addition to PDGF-BB will be important to define the secretory function of this cell type within the bone marrow microenvironment under disease conditions.

Our data suggest that PDGF-BB exerts paradoxical bone effects depending on the concentration of PDGF-BB in the bone microenvironment. We previously showed that a normal range of PDGF-BB is essential for the maintenance of bone homeostasis in young, healthy mice because deletion of $P d g$ $f b$ from $\mathrm{TRAP}^{+}$preosteoclasts led to reduced trabecular and cortical bone mass (28). Consistently, here we also found a low-bone-mass phenotype in the young, healthy Pdgfb-cKO mice relative to their WT littermates. However, during aging or under metabolic stress conditions such as HFD challenge, aberrantly elevated PDGF-BB in bone marrow microenvironment exerts an adverse bone effect. Importantly, deletion of $P d g f b$ in preosteoclasts normalized the PDGF-BB concentration and largely rectified the pathological bone phenotype induced by aging and HFD. Furthermore, the young Pdgfb ${ }^{\mathrm{cTG}}$ mice, resemblant of the old mice, have a much-elevated PDGF-BB concentration in bone marrow. As a result, the mice develop an aging-associated trabecular bone loss phenotype. Together, our finding imply that PDGF-BB is required for the maintenance of bone homeostasis under normal physiological conditions, and aberrantly elevated PDGF-BB in bone marrow microenvironment leads to bone loss in pathological conditions (e.g., aging and metabolic dysregulation). At the cellular level, we found a substantially reduced osteoblast number but an unchanged osteoclast number in the trabecular compartment of long bone in the Pdgfb ${ }^{\mathrm{TTG}}$ mice relative to WT mice. The data suggest that excessive PDGF-BB produced by preosteoclasts negatively regulates osteoblast differentiation activity in a paracrine manner, leading to impaired bone formation.

Our results further reveal that skeletal preosteoclasts are a main cell type contributing to the elevation of PDGF-BB in blood circulation during aging and under metabolic stress. While aged mice and those fed HFD had higher serum PDGF-BB relative to young mice and CHD mice respectively, mice with $P d g f b$ deletion from preosteoclasts had a normalized serum PDGF-BB concentration. Moreover, young conditional Pdgf $b$ transgenic mice, resemblant of the aged mice, had a much higher serum PDGFBB level relative to their WT littermates. Therefore, skeletal preosteoclast-derived PDGF-BB is both sufficient and required to cause circulating PDGF-BB elevation. We are aware that the increased PDGF-BB may also be produced from tissues other than bone. However, we detected increased expression of $P d g$ $f b$ mRNA and PDGF-BB protein only in preosteoclasts but not in the periphery blood myeloid cells and aorta tissue from Pdgfb ${ }^{\mathrm{cTG}}$ mice relative to WT mice. The results suggest that the elevated circulating PDGF-BB in response to aging or metabolic stress is primarily produced by bone/bone marrow preosteoclasts rather than a local effect derived from arterial vessel wall or blood myeloid cells. We are aware that the Trap-Cre line causes deletion of PDGF-BB in all Trap ${ }^{+}$osteoclast lineage cells, including 

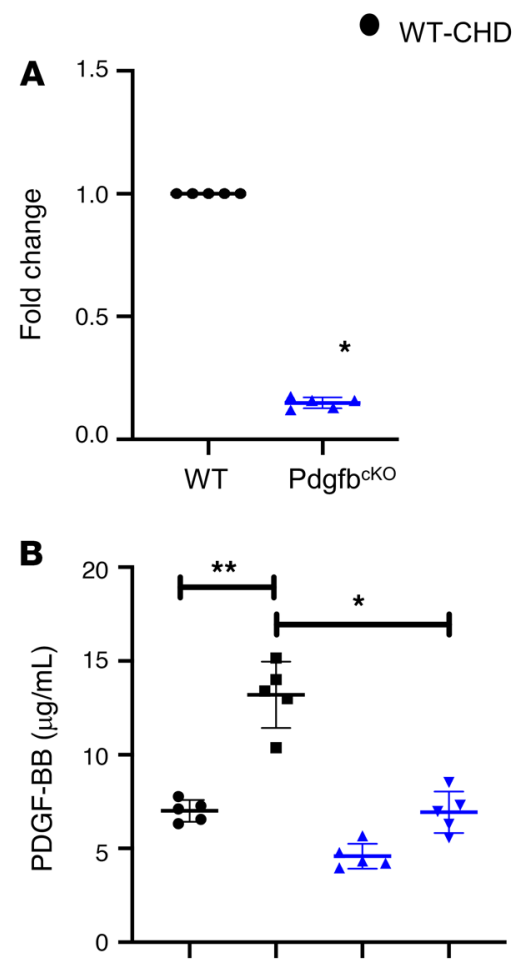

WT-HFD

C

CHD
WT

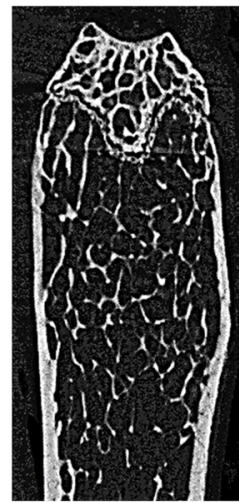

H
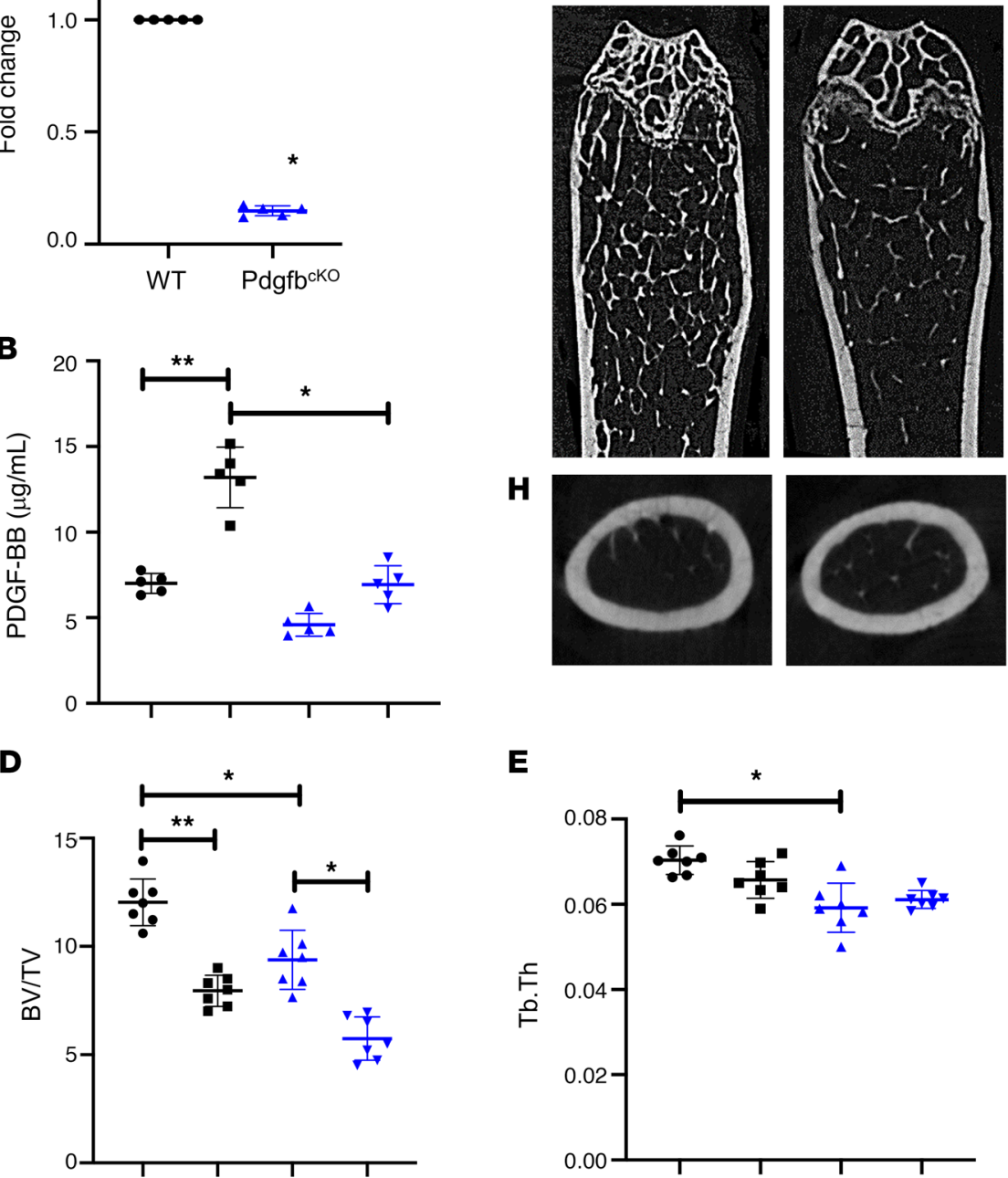

$\mathbf{G}$

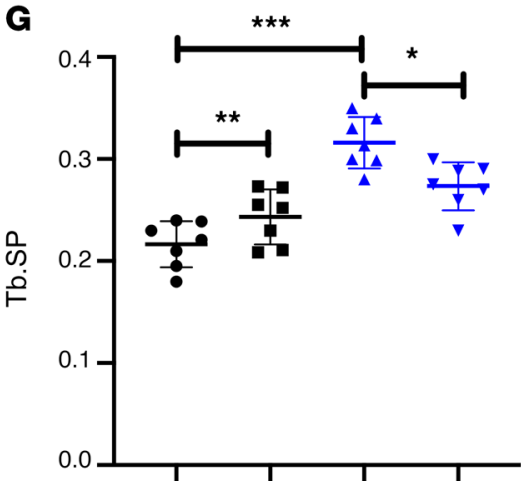

E

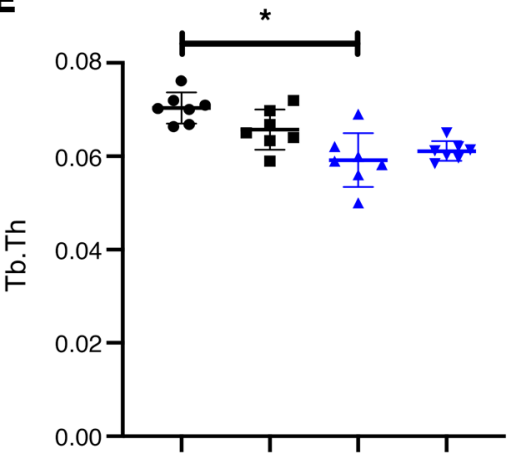

I

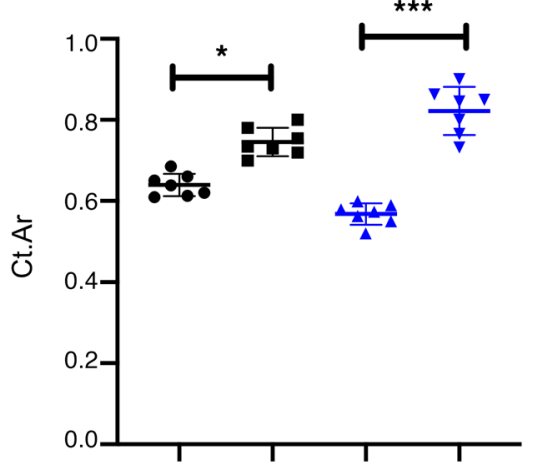

Pdgfb-cKO-HFD

HFD

WT
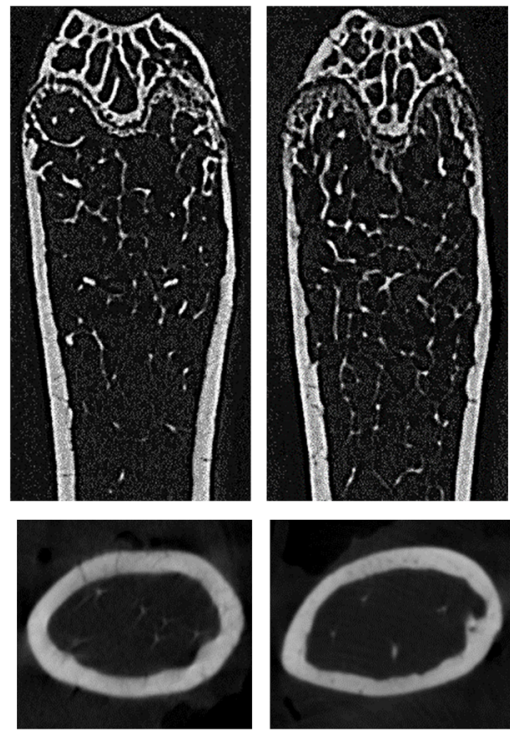

$\mathbf{F}$

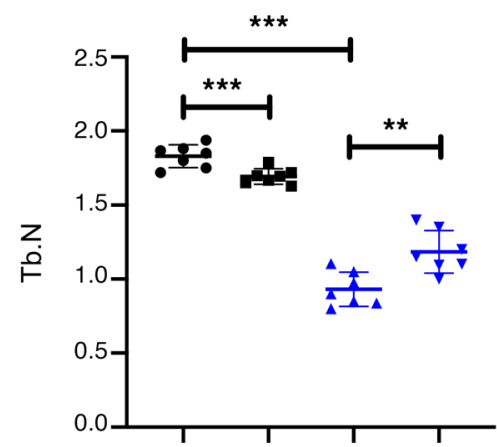

J

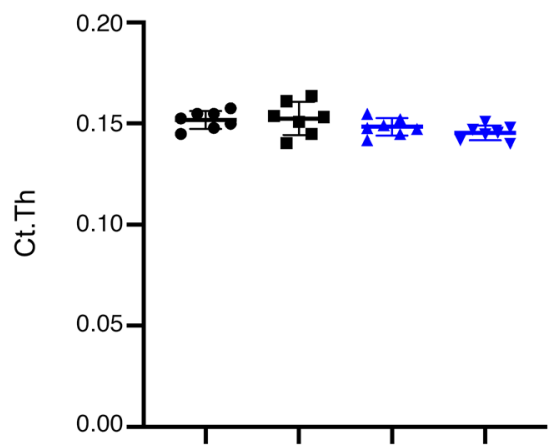

Figure 10. Deletion of Pdgfb in preosteoclasts attenuates HFD-induced bone loss. (A) Measurement of PDGF-BB mRNA in isolated CD3/B220/T119$\mathrm{RANK}^{+}$cells from bone/bone marrow of Trap-Cre Pdgfbf/f mice (Pdgfb-cKO) and Pdgfbf/f littermates (WT). (B) ELISA measurements of serum PDGF-BB concentrations in Pdgfb-cKO and WT littermates fed a HFD or CHD. $n=5$. Data are mean \pm SD. ${ }^{*} P<0.05$, ${ }^{*} P<0.01$, as determined by Student's $t$ tests and 1-way ANOVA with Bonferroni post hoc test. (C-C) Representative $\mu \mathrm{CT}$ images (C) and quantitative analysis of the trabecular bone area of the distal femur from 3-month-old Pdgfb-cKO mice and WT littermates after 14 weeks of a Western HFD or chow diet (CHD). BV/TV (D), Tb.Th (E), Tb.N (F), and Tb.Sp (G). Representative $\mu \mathrm{CT}$ images $(\mathbf{H})$ and quantitative analysis (I and $\mathbf{J})$ of the cross-sections of femoral mid-diaphysis of mice. $n=7$. Data are mean \pm SD. ${ }^{*} P<0.05,{ }^{* *} P<0.01,{ }^{* * *} P<0.001$, as determined by 1-way ANOVA with Bonferroni post hoc analysis. 
$\mathrm{TRAP}^{+}$mononuclear preosteoclasts and $\mathrm{TRAP}^{+}$multinuclear mature osteoclasts. However, PDGF-BB is secreted specifically by $\mathrm{Trap}^{+}$mononucleate preosteoclasts, and a very low level of PDGF-BB is secreted by mature osteoclasts (ref. 25 and Figure 4). Moreover, our data from the TRAP/tdTom mice clearly demonstrate that although there is a nonspecific Cre expression, cells in aortic tissue do not produce PDGF-BB. Therefore, the effect of local aorta tissue-produced PDGF-BB can be excluded by using the TRAP-Cre line, and the reduced PDGF-BB concentration in the Trap-Cre-driven knockout mice is caused mainly by the deletion of PDGF-BB from preosteoclasts.

PDGF-BB overexpression accelerated aging-associated vascular stiffening. A well-known VSMC mitogen, PDGF-BB can elicit VSMC migration and proliferation in the aging aorta, leading to structural/compositional changes that are characteristic of the aged, stiff vessel. For example, aging VSMCs express higher levels of PDGF-BB receptor (57) and exhibit augmented proliferation in response to PDGF-BB $(76,77)$. Thus, PDGF-BB can cause diffuse intimal changes in the aging vessel, which is a hallmark of aging (78). In this study, we show that PDGF-BB secreted by preosteoclasts contributes to vascular aging. In vitro, conditioned media from preosteoclasts overexpressing $P d g f b$ promoted VSMC proliferation. In vivo, young Pdgfb ${ }^{\mathrm{cTG}}$ mice have stiffer vessels as reflected by the augmented PWV, and higher systolic and diastolic BPs. The passive stiffness of the vessels measured by tensile testing shows a strikingly higher stiffness of the young Pdgfb ${ }^{\mathrm{cTG}}$ mice compared with their littermates. The augmented PWV is likely to be a combination of increased BP and passive stiffening in the conditional transgenic mice. Notably, while the passive stiffness of WT mice increased remarkably with age, this did not occur in the Pdgfb ${ }^{\text {cTG }}$ mice, in which the young vessels were markedly stiffer to begin with. Moreover, with age, there was a marked increase in PWV, systolic BP, and diastolic BP in the WT mice, but not in the Pdgfb ${ }^{\mathrm{cT}}$ mice. At the histology level, Pdgfb ${ }^{\mathrm{cTG}}$ mice exhibited increased collagen fibers in the extracellular matrix and calcification of the aorta, suggesting that vascular fibrosis and calcification are major contributors to PDGFB/PDGFR $\beta$-associated arterial stiffening. Interestingly, aging resulted in a higher PWV in female $\mathrm{Pdgfb}^{\mathrm{cTG}}$ mice when compared with age-matched males, while BP was not notably different between the sexes. This is an intriguing finding, particularly in the context of higher incidence of osteoporosis in elderly females. We previously noted that in rodents, the stiffening trajectory is to have a steep increase in stiffness between 3 to 12 months, and then a plateau of values (79). Considering these prior reports, we postulate that Pdgfb ${ }^{\mathrm{cTG}}$ mice have an accelerated vascular stiffening and reach a plateau much earlier in their lifespan than do the WT mice. Therefore, when we compare the aged ( $>18$ months old) WT and $\mathrm{Pdgfb}^{\mathrm{cTG}}$ mice, the differences are no longer as remarkable because both cohorts have reached the maximal plateau.

Accumulating evidence points to VSMC stiffening and tone as key mediators of overall vascular stiffness. This is particularly interesting in this study, as PDGF-BB also acts as a vasoconstrictor $(75,80,81)$. Our study further shows that PDGF-BB contributes to the functional contractility of VSMCs because aging resulted in a marked sensitization of the agonist-induced vasoconstriction response and an attenuated vasorelaxation response in the $\mathrm{Pdgfb}^{\mathrm{cTG}}$ mice. Conversely, reduced levels of cir- culating PDGF-BB attenuated phenylephrine contractility and augmented acetylcholine-induced vasorelaxation in the Pdgfb ${ }^{\text {cTG }}$ mice. These findings are also consistent with global knockout of the Pdgfb gene, which showed a loss of functional contractility of VSMCs, causing a remarkable dilation of the aorta rather than producing structural deficits in the large compliance vessels (82). In young Pdgfb ${ }^{\text {cTG }}$ mice, despite the vascular stiffening noted, there is no compromised contraction or endothelial relaxation response. Prior studies have shown that PDGF-BB caused severe and chronic vasoconstriction (83). Interestingly, however, we did not note a change in contraction responses in the young mice. One reason for the unchanged contraction or endothelial relaxation response in young transgenic mice may be that PDGF receptor is not expressed highly in the young vessels. However, in the old Pdgfb ${ }^{\mathrm{cTG}}$ mice, a significantly higher contraction response was noted. This is likely due to increased expression of PDGF-R in the aged vessels (Figure 8J) in good agreement with the literature (84). We also found more VSMC nuclei loss in the aged WT mice than in the aged Pdgfb ${ }^{\mathrm{cTG}}$ mice. This can also be attributed to the upregulated PDGF-R expression and Pdgfb signaling that may lead to VSMC proliferation in the old conditional transgenic mice. Our studies further show that the lumen diameter of the aged $\mathrm{Pdgfb}^{\mathrm{cTG}}$ mice is higher than the WT littermates. Furthermore, while wall thickness was not significantly higher in the aged $\mathrm{Pdgfb}^{\mathrm{cTG}}$ mice, the number of cells in the vessel wall was higher. Collectively, our findings from young and old Pdgfb ${ }^{\mathrm{cTG}}$ mice imply that conditional Pdgfb transgenic mice not only have an accelerated stiffening response to age, but also exaggerated endothelial and vascular dysfunction. More importantly, arterial stiffening induced by a HFD was significantly alleviated in the PDGF-BB knockout mice, confirming the critical role of preosteoclast-derived PDGF-BB in the increase in vascular stiffness. Thus, our study supports a growing body of evidence showing that in addition to the extracellular matrix stiffness and remodeling, aortic tone and VSMC stiffness are critical determinants of in vivo vascular stiffness.

The observation that young conditional $P d g f b$ transgenic mice fed a CHD develop both low bone mass and an arterial stiffening phenotype spontaneously is intriguing. This phenomenon implies that preosteoclast-derived increases in PDGF-BB, which are sufficient to cause bone loss and arterial stiffening, function as a molecular link for the bone/vascular axis. Of great relevance for the clinic, our finding reveals that serum PDGF-BB can be used as a biomarker to determine who are at the greatest risk for age-associated vascular diseases. Our observations also provide the basis for future investigations to determine whether targeting preosteoclasts or PDGF-BB signaling is an efficient strategy to prevent or treat cardiovascular disease in the elderly population, especially those who develop cardiovascular disease together with osteoporosis. The use of intervention to inhibit or neutralize PDGF-BB in the aging and under HFD conditions is a goal of future studies.

\section{Methods}

Animals and treatment. Male C57BL/6J mice were purchased from The Jackson Laboratory. Male Sprague-Dawley rats were purchased from Charles River Laboratories. All animals were bred and housed in the 
A

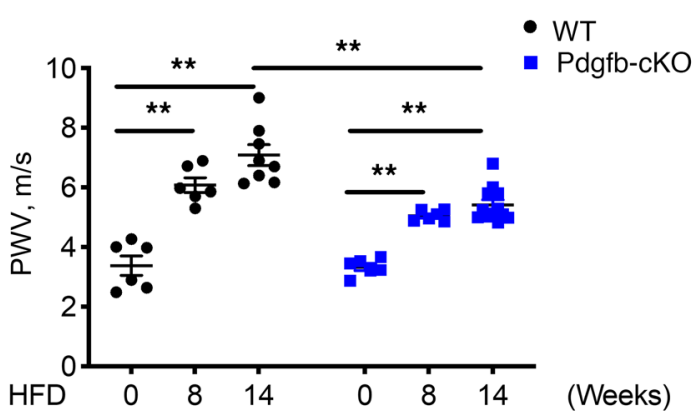

B

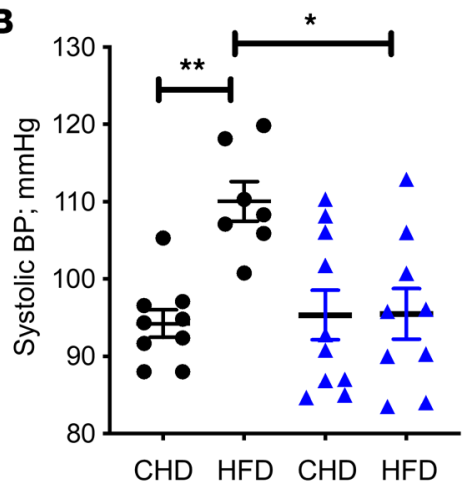

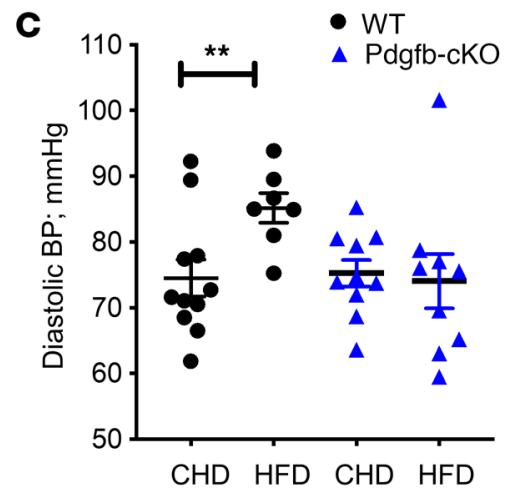

$\mathbf{F}$

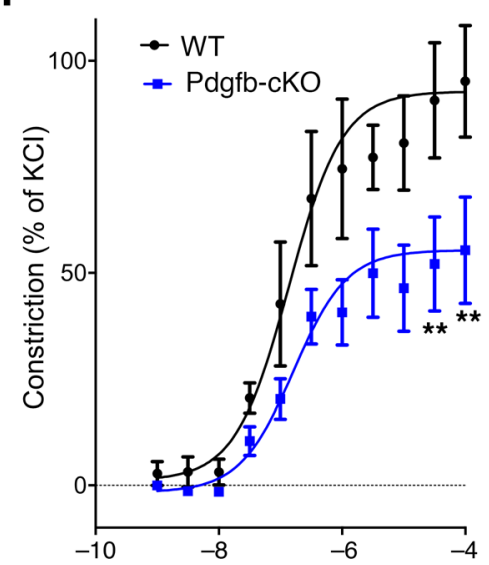

G

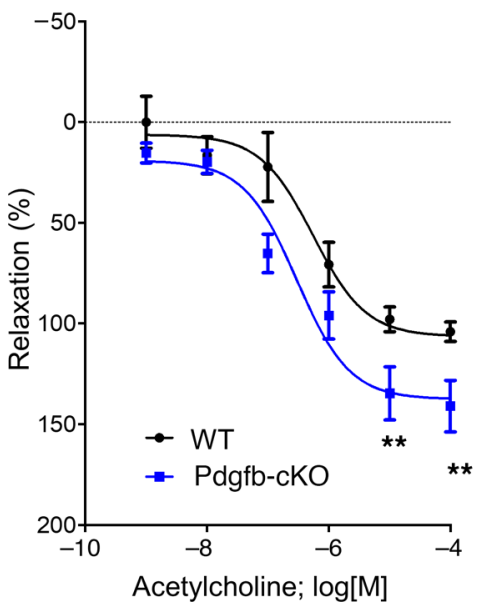

E

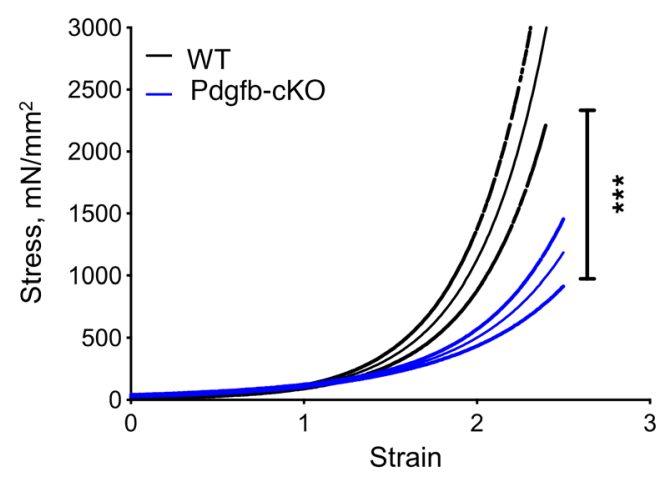

H

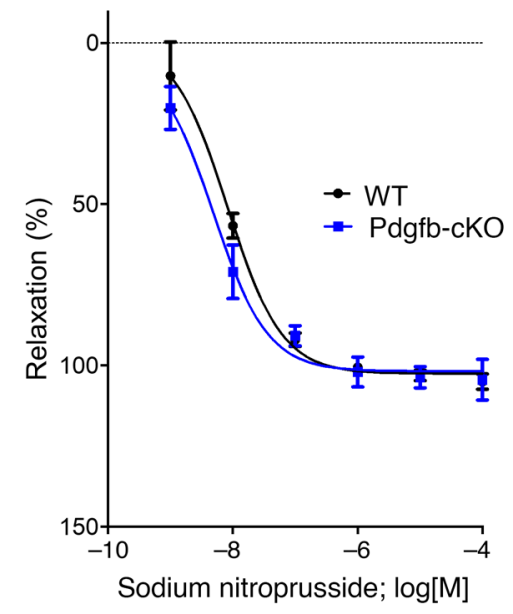

Figure 11. Deletion of Pdgfb in preosteoclasts attenuates HFD-induced vascular stiffening. PWV (A), systolic BP (B), diastolic BP (C), and mean BP (D) were measured in Pdgfb-cKO and WT littermates after 8 and 14 weeks of a Western HFD. $n=6$ to 11. Data are mean \pm SEM. ${ }^{*} P<0.05,{ }^{* *} P<0.01$ versus WT mice, as determined by 1-way ANOVA with Bonferroni post hoc test. (E) Tensile testing of aortic rings was measured after 14 weeks of a Western HFD diet. $n=10$ rings. Data are mean \pm SEM. ${ }^{* * *} P<0.001$ versus WT mice, as determined by 1 -way ANOVA with Bonferroni post hoc analysis. (F) Phenylephrine-induced contractility was measured after 14 weeks of a HFD in Pdgfb-cKO and WT littermates. $n=8$ to 10 rings. Data are mean \pm SEM. ${ }^{* *} P<0.01$, as determined by 1-way ANOVA with Bonferroni post hoc analysis. (G) Acetylcholine-induced endothelium-dependent relaxation of phenylephrine-preconstricted aortic rings was measured after 14 weeks of a HFD in Pdgfb-cKO and WT littermates. $n=8$ to 10 rings. Data are mean \pm SEM. ${ }^{* *} P<0.01$, as determined by 1-way ANOVA with Bonferroni post hoc analysis. (H) The endothelial-independent sodium nitroprusside-induced relaxation was measured after 14 weeks of a HFD in Pdgfb-cKO and WT littermates. 


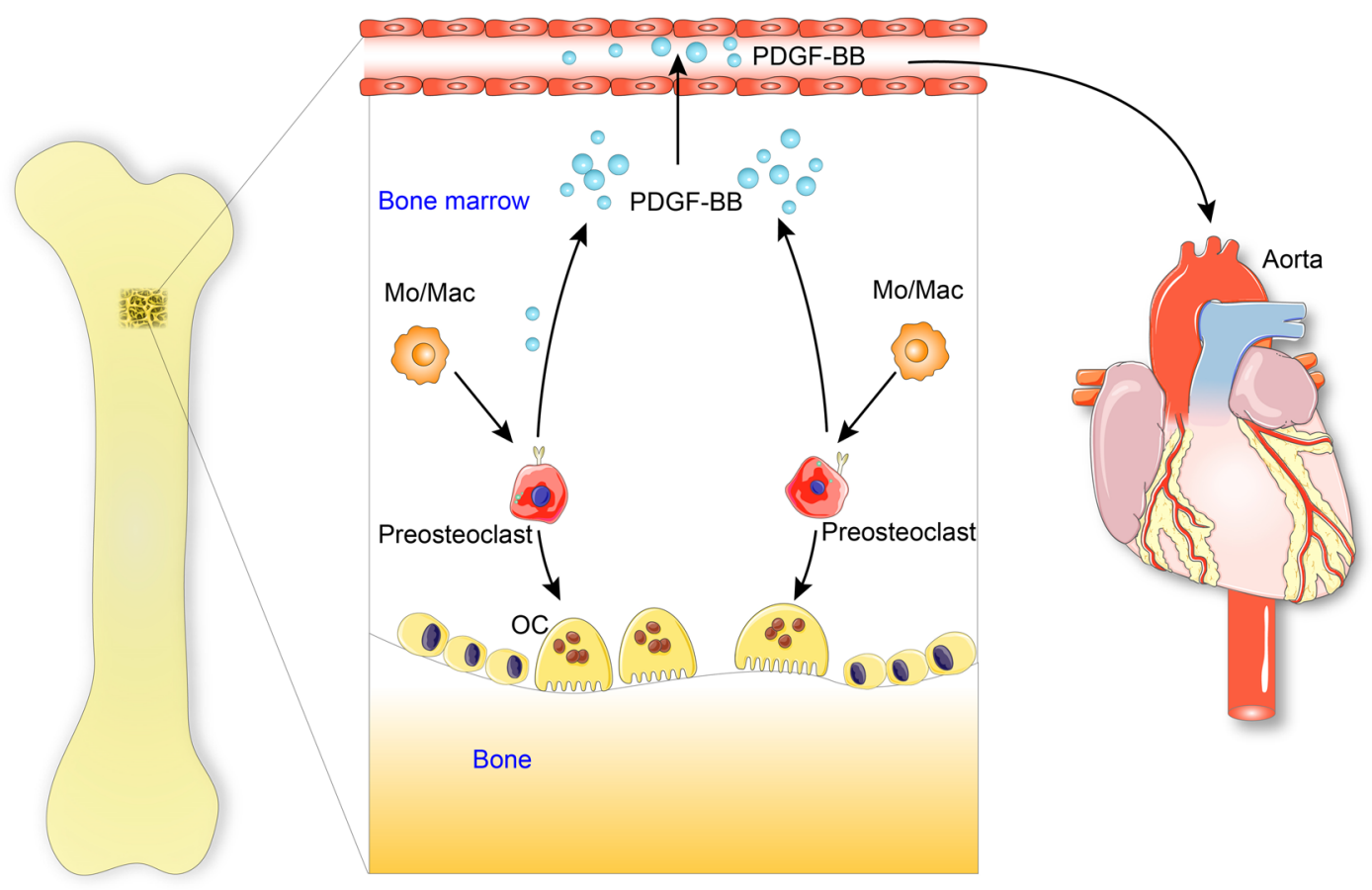

Figure 12. Schematic model illustrating the bone-vascular interplay during aging. With advancing age or under metabolic stress, preosteoclasts in bone secrete a high amount of PDGF-BB, which infuses into blood circulation. Elevated circulating PDCF-BB, serving as a systemic progeronic factor, drives arterial stiffening.

animal facility at our institution. At 10 to 12 weeks of age, mice were placed on a Western HFD (21.2\% fat by weight) (TD 88137, Harlan Laboratories) or normal CHD for 8 to 20 weeks.

Micro-CT and histomorphometric analyses of femoral bone. Mice were anesthetized by inhalation of $2.5 \%$ isoflurane (Abbott Laboratories) mixed with $\mathrm{O}_{2}(1.5 \mathrm{~L} / \mathrm{min})$. For $\mu \mathrm{CT}$ analysis, mice femora were dissected free of soft tissue, fixed overnight in $10 \%$ formalin at $4^{\circ} \mathrm{C}$, and analyzed by high-resolution $\mu \mathrm{CT}$ (Skyscan 1172, Bruker MicroCT). The scanner was set at $65 \mathrm{kV}, 153 \mu \mathrm{A}$, and a resolution of $9.0 \mu \mathrm{m} /$ pixel. We used NRecon image reconstruction software, version 1.6 (Bruker MicroCT), CTAn data analysis software, version 1.9 (Bruker MicroCT), and CTVol 3D model visualization software, version 2.0 (Bruker MicroCT) to analyze parameters of trabecular bone in the metaphysis. To perform 3D histomorphometric analysis of trabecular bone, we selected the regions of interest from $1 \mathrm{~mm}$ below the distal epiphyseal growth plate and extended distally for proximally 2 $\mathrm{mm}$. Trabecular bone was analyzed to determine trabecular BV/TV, Tb.Th, Tb.N, and Tb.Sp. Cortical morphometry was analyzed within a $600 \mu \mathrm{m}$ long section at mid-diaphysis of the femur and included measurements of average thickness and cross-sectional area.

For histomorphometric analysis, the femora were resected and fixed in $4 \%$ paraformaldehyde for 48 hours, decalcified in 0.5M EDTA (pH 7.4) at $4^{\circ} \mathrm{C}$, and embedded in paraffin. Five $\mu \mathrm{m}$-thick longitudinally oriented sections of bone were processed for OCN immunohistochemical staining (for osteoblast analysis), and TRAP staining (for osteoclast analysis). All sections were observed using an Olympus BX51 microscope. Quantitative histomorphometry analyses were performed as described previously (85) in a blinded fashion using OsteoMeasure Software (OsteoMetrics, Inc.). The sample area selected for calculation was a $1 \mathrm{~mm}^{2}$ area within the metaphyseal trabecular bone. Number of osteoblasts per bone perimeter (N.OB/B.Pm) and number of osteoclasts per bone perimeter (N.OC/B.Pm) in 5 randomly selected visual fields per specimen, in 4 specimens per mouse in each group were measured.

Immunofluorescence staining of bone and aorta tissue sections. Femora and aortas were dissected after mouse sacrifice and fixed in PBS $(\mathrm{pH}$ 7.4) containing $4 \%$ paraformaldehyde for 48 hours. Femora were then decalcified in 0.5M EDTA ( $\mathrm{pH}$ 7.4) with constant shaking for 8 days. For dehydration, the decalcified bones and aortas were immersed in a solution of $20 \%$ sucrose and $2 \%$ polyvinylpyrrolidone for 24 hours. The tissues were embedded in OCT, and $10 \mu \mathrm{m}$-thick longitudinally oriented sections of bone were collected for immunofluorescence staining as previously described (86). Transverse and longitudinal sections of aorta were also prepared. The tissue sections were incubated with primary antibody to PDGF-BB (ab178409, Abcam, 1:50, polyclonal) or PDGFR $\beta$ (ab32570, Abcam, 1:100, monoclonal) followed by fluorescence-conjugated secondary antibodies. Nuclei were counterstained with DAPI (Sigma-Aldrich). The sections were mounted with the ProLong Antifade Kit (Molecular Probes) and observed under a Zeiss LSM 780 confocal microscope (Carl Zeiss AG).

Isolation of bone/bone marrow preosteoclasts and blood myeloid cells. To isolated bone/bone marrow preosteoclasts, 2 approaches were used. As the first approach, a mixture of bone and bone marrow cell suspensions was prepared from C57B/L6 mice as previously described (86), with modifications. Briefly, the epiphysis was removed from the distal femora and proximal tibia, and bone marrow was flushed. Moreover, the metaphyseal region of bone tissue was harvested, crushed in ice-cold PBS with a mortar and pestle, and digested with collagenase I ( $3 \mathrm{mg} / \mathrm{mL})$, dispase I $(4 \mathrm{mg} / \mathrm{mL})$, and deoxyribonuclease $(1 \mathrm{U} / \mathrm{mL})$ in $\mathrm{PBS}$ at $37^{\circ} \mathrm{C}$ for 30 minutes. The resultant single bone cell suspensions were combined with the flushed bone marrow 
cells. After red cell lysis, cells were incubated with a mix of antibodies against CD3 (a T cell marker, ab16669, Abcam), CD45R/B220 (a B cell marker, 562313, Becton Dickinson), and Ter119 (an erythrocytes marker, 14-5921-82, Thermo Fisher Scientific), all conjugated to APC. After exclusion of CD3/B220/Ter119 ${ }^{+}$cells, RANK $^{+}$cells were sorted using CD265 (RANK) antibody (119805, BioLegend) by FACS. As the second approach, a mixture of bone and bone marrow cell suspensions was prepared from TRAP/tdTom mice as described above. After exclusion of CD3/B220/Ter119+ cells, tdTom $^{+}$cells were sorted by FACS using a 5-laser FACS and FACS Diva (Becton Dickinson). To isolate periphery blood myeloid cells, EasySep Mouse Monocyte Isolation Kit (STEMCELL Technologies Inc.) was used following manufacturer's instructions.

Human subjects and ELISA analysis of serum PDGF-BB concentration. Serum samples collected from a total of 15 human participants were included. The "young" cohort was composed of individuals 21 to 26 years old. Three were female and 2 were male. The "old" cohort was composed of individuals 58 to 71 years old. Six were female (post-menopause) and 4 were male. All participants were healthy with no reported comorbidities of obesity, diabetes, heart disease, hypertension, and other vascular disorders.

$P W V$ measurement. In vivo vascular stiffness was analyzed by obtaining invasive PWV measurements at mean arterial pressures varying from 55 to $130 \mathrm{~mm} \mathrm{Hg}$ as previously described $(13,14,26)$. We used a high-fidelity dual-pressure catheter sensor to measure aortic PWV. Mice were anesthetized with an intraperitoneal injection of $1.2 \%$ Avertin (2,2,2-tribromoethanol, $240 \mathrm{mg} / \mathrm{kg}$ ) in $0.9 \%$ saline. The mouse was positioned supine on the heating pad, with water temperature set to $40^{\circ} \mathrm{C}$. Anesthesia was maintained by mask ventilation with $1.0 \%$ to $1.5 \%$ isoflurane (in $100 \% \mathrm{O}_{2}$ ), and the reflex response to hindpaw pinching was assessed to monitor depth of anesthesia. After making a midline neck incision from mandible to sternum, we introduced a 1.2-Fr, dual-pressure sensor catheter (Scisense) into the descending thoracic aorta through the left carotid artery without opening the chest cavity. The distance between 2 sensors is fixed at $1 \mathrm{~cm}$. A 30-gauge cannulation needle connected to polyethylene tubing was inserted into the left femoral vein for infusion of fluid/drugs. After stabilization of the signal for 10 to 15 minutes, baseline blood pressures were recorded. Mean arterial pressure was raised and lowered to obtain a full physiological range of blood pressure using intravenous infusion of phenylephrine and sodium nitroprusside, respectively. PWV at corresponding mean arterial pressure was calculated using the foot-tofoot method, the foot being defined by the peak of the second time derivative of 2 aortic pressures measured simultaneously during each pulse. PWV was plotted against mean arterial pressure to construct phase plots to characterize PWV over a wide range of mean arterial pressures from 50 to $150 \mathrm{~mm} \mathrm{Hg}$ in the aorta.

Noninvasive blood pressure measurements. Blood pressure of awake mice was determined by a tail cuff measurement system as described previously (Kent Scientific) $(26,60)$. Systolic, mean, and diastolic BPs were measured.

Tensile testing. The elastic properties of the samples were analyzed by tensile testing as previously described $(13,26)$. We used the descending aortas of mice, which were harvested and cut into 2-mm rings. The sample to be tested was imaged longitudinally, and the cross-section of a $0.5-\mathrm{mm}$ segment proximal to the test sample ring was imaged at $\times 10$ magnification along with a graticule. Vessel lumen diameter $\left(D_{i}\right)$, wall thickness $(t)$, and sample length were calculated using Image J software (NIH). The 2-mm ring was then mounted onto the pins of an electromechanical puller (DMT560; Danish Myo Technology A/S). After calibration and alignment, the pins were slowly moved apart using an electromotor at a rate of $50 \mu \mathrm{m} / \mathrm{s}$ to apply radial stress on the specimen until breakage. Displacement and force were recorded continuously. Engineering stress (S) was calculated by normalizing force $(\mathrm{F})$ to the initial stress-free area of the specimen using the following equation: $\mathrm{S}=\mathrm{F} / 2 \mathrm{t} \times \mathrm{l}$, in which $\mathrm{t}=$ thickness and $\mathrm{l}=$ length of the sample. Engineering strain $(\lambda)$ was calculated as the ratio of displacement to the initial stress-free diameter. The stress-strain relationship was represented by the following equation: $S=\alpha \exp (\beta \lambda)$, in which $\alpha$ and $\beta$ are constants. $\alpha$ and $\beta$ were determined by nonlinear regression for each sample and used to generate stress-strain curves by treating the $x$ axis as a continuous variable.

Wire myography. Vasoconstriction in response to phenylephrine treatment was examined by wire myography as previously described $(13,26)$. Briefly, after careful excision and cleaning from the surrounding soft tissues, the thoracic aorta was cut into 2-mm rings. The endothelium was removed by mechanical scraping for a subset of the rings. Each ring was placed in Krebs (containing [in mmol/L] 118.3 NaCl, 4.7 $\mathrm{KCl}, 1.6 \mathrm{CaCl}_{2}, 1.2 \mathrm{KH}_{2} \mathrm{PO}_{4}, 25 \mathrm{NaHCO}_{3}, 1.2 \mathrm{MgSO}_{4}$, and 11.1 dextrose at a $\mathrm{pH}$ of 7.4) and then transferred to a myograph chamber (DMT) and continuously bubbled with $95 \% \mathrm{O}_{2}$ and $5 \% \mathrm{CO}_{2}\left(37^{\circ} \mathrm{C}\right)$. The rings were stretched in 100-mg increments to a final tension of $600 \mathrm{mg}$. After passive stretching of the rings, $\mathrm{KCl}(60 \mathrm{mmol} / \mathrm{L})$ was added to determine the viability of the vascular preparation and to obtain maximal contractility. Concentration-response curves were constructed for phenylephrine $\left(10^{-9}\right.$ to $\left.10^{-5} \mathrm{~mol} / \mathrm{L}\right)$. Next, endothelial-mediated vasorelaxation was studied using increasing doses of acetylcholine $\left(10^{-9}\right.$ to $10^{-5} \mathrm{~mol} / \mathrm{L}$ ) in vessels preconstricted with phenylephrine. Finally, endothelial-independent vasorelaxation mediated by increasing doses of sodium nitroprusside $\left(10^{-9}\right.$ to $\left.10^{-5} \mathrm{~mol} / \mathrm{L}\right)$ was examined in vessels preconstricted with phenylephrine.

Aorta histology, von Kossa staining, and quantitative analysis. Descending thoracic aortas from mice were harvested and fixed in $10 \%$ formalin for 48 hours and then transferred to $70 \%$ ethanol for storage at $4^{\circ} \mathrm{C}$. Fixed aortic segments were embedded in paraffin and sectioned at $5 \mu \mathrm{m}$ thickness. Sequential sections were stained with hematoxylin and eosin as well as Masson's trichrome staining. Images were acquired using an Olympus BX51 microscope. Aortic diameter and wall thickness were determined by $10 \times$ images using ImageJ. Number of cells in the vascular media was determined using object count in ImageJ. Calcification of the descending thoracic aortas was detected by von Kossa staining of $5-\mu \mathrm{m}$ longitudinal cryosections as previously described (87). Calcification was visualized as distinct black deposits of calcium using an BX51 microscope (Olympus) coupled with imaging software (Cellsens). Additional methods are described in the Supplemental Material (see supplemental material; available online with this article; https://doi.org/10.1172/JCI147116).

Statistics. Data are presented as mean \pm standard error. Unpaired, 2 -tailed Student's $t$ tests were used for comparisons between 2 groups. For multiple comparisons, 1-way analysis of variance (ANOVA) with Bonferroni post hoc test was used. All data were normally distributed and had similar variation between groups. Statistical analyses were performed using SAS software, version 9.3 (SAS Institute) or GraphPad Prism 9.0. $P$ less than 0.05 was deemed significant. 
Study approval. All animal studies described were conducted under protocol MO18M139, approved by the IACUC of The Johns Hopkins University, Baltimore, Maryland. The study protocol for human serum sample analysis under protocol IRB00251934 was approved by the IRBs at The Johns Hopkins University. Procedures were followed in accordance with institutional committees on human experimentation.

\section{Author contributions}

LS and MW designed the experiments. LS, GL, SJ, W Su, BPW, W Savage, AP, and XL carried out the experiments. LMA collected the human serum samples. XC proofread the manuscript. LS and MW supervised the experiments, analyzed results, and wrote the manuscript.

\section{Acknowledgments}

The authors acknowledge the assistance of Jenni Weems, Rachel Box, and Kerry Kennedy at The Johns Hopkins Department of Orthopaedic Surgery Editorial Services for editing the manuscript. This work was supported by National Institutes of Health grants R01 AG068226 and R01AG072090 (to MW), R01HL148112 (to LS), and P01AG066603 (to XC).

Address correspondence to: Lakshmi Santhanam, Ross Building, Room 1150, 720 Rutland Avenue, Baltimore, Maryland 21205, USA. Email: 1santha1@jhmi.edu. Or to: Mei Wan, Ross Building, Room 232, 720 Rutland Avenue, Baltimore, Maryland 21205, USA.Email: mwan4@jhmi.edu.
1. Fadini GP, et al. Emerging role of circulating calcifying cells in the bone-vascular axis. Circulation. 2012;125(22):2772-2781.

2. Khosla S. The bone and beyond: a shift in calcium. Nat Med. 2011;17(4):430-431.

3. Shao JS, et al. Molecular mechanisms of vascular calcification: lessons learned from the aorta. Arterioscler Thromb Vasc Biol. 2006;26(7):1423-1430.

4. Thompson B, Towler DA. Arterial calcification and bone physiology: role of the bone-vascular axis. Nat Rev Endocrinol. 2012;8(9):529-543.

5. Byon $\mathrm{CH}$, Chen Y. Molecular mechanisms of vascular calcification in chronic kidney disease: the link between bone and the vasculature. Curr Osteoporos Rep. 2015;13(4):206-215.

6. Tanko LB, et al. Low bone mineral density in the hip as a marker of advanced atherosclerosis in elderly women. Calcif Tissue Int. 2003;73(1):15-20.

7. Tanko LB, et al. Relationship between osteoporosis and cardiovascular disease in postmenopausal women. J Bone Miner Res. 2005;20(11):1912-1920.

8. Hyder JA, et al. Bone mineral density and atherosclerosis: the Multi-Ethnic Study of Atherosclerosis, Abdominal Aortic Calcium Study. Atherosclerosis. 2010;209(1):283-289.

9. Kovacic JC, Fuster V. Vascular calcification, diabetes, and cardiovascular disease: connecting the dots. JACC Cardiovasc Imaging. 2012;5(4):367-369.

10. Parhami F, et al. Atherogenic high-fat diet reduces bone mineralization in mice. J Bone Miner Res. 2001;16(1):182-188.

11. Towler DA, et al. Osteogenic regulation of vascular calcification. Ann N Y Acad Sci. 2006;1068:327-333.

12. Farhat GN, Cauley JA. The link between osteoporosis and cardiovascular disease. Clin Cases Miner Bone Metab. 2008;5(1):19-34.

13. Sambrook PN, et al. High bone turnover is an independent predictor of mortality in the frail elderly. J Bone Miner Res. 2006;21(4):549-555.

14. Elmariah S, et al. Bisphosphonate use and prevalence of valvular and vascular calcification in women MESA (The Multi-Ethnic Study of Atherosclerosis). J Am Coll Cardiol. 2010;56(21):1752-1759.

15. Demer LL, Tintut Y. Interactive and multifactorial mechanisms of calcific vascular and valvular disease. Trends Endocrinol Metab. 2019;30(9):646-657.

16. Tintut $\mathrm{Y}$, et al. Lipoproteins in cardiovascular cal- cification: potential targets and challenges. Front Cardiovasc Med. 2018;5:172.

17. Hsu JJ, et al. Cell-matrix mechanics and pattern formation in inflammatory cardiovascular calcification. Heart. 2016;102(21):1710-1715.

18. Guntur AR, Rosen CJ. Bone as an endocrine organ. Endocr Pract. 2012;18(5):758-762.

19. Karsenty G, Oury F. Biology without walls: the novel endocrinology of bone. Annu Rev Physiol. 2012;74:87-105.

20. Drissi H, Sanjay A. The multifaceted osteoclast; far and beyond bone resorption. JCell Biochem. 2016;117(8):1753-1756.

21. Cappariello A, et al. The great beauty of the osteoclast. Arch Biochem Biophys. 2014;558:70-78.

22. Teti A. Mechanisms of osteoclast-dependent bone formation. Bonekey Rep. 2013;2:449.

23. Boyce BF. Advances in the regulation of osteoclasts and osteoclast functions. J Dent Res. 2013;92(10):860-867.

24. Yavropoulou MP, Yovos JG. Osteoclastogenesis current knowledge and future perspectives. JMusculoskelet Neuronal Interact. 2008;8(3):204-216.

25. Ishii M, Saeki Y. Osteoclast cell fusion: mechanisms and molecules. Mod Rheumatol. 2008;18(3):220-227.

26. Manolagas SC. Birth and death of bone cells: basic regulatory mechanisms and implications for the pathogenesis and treatment of osteoporosis. Endocr Rev. 2000;21(2):115-137.

27. Henriksen K, et al. Osteoclast activity and subtypes as a function of physiology and pathology -implications for future treatments of osteoporosis. Endocr Rev. 2011;32(1):31-63.

28. Xie H, et al. PDGF-BB secreted by preosteoclasts induces angiogenesis during coupling with osteogenesis. Nat Med.2014;20(11):1270-1278.

29. $\mathrm{Su} \mathrm{W}$, et al. Angiogenesis stimulated by elevated PDGF-BB in subchondral bone contributes to osteoarthritis development. JCI Insight. 2020;5(8):e135446.

30. Kohn JC, et al. Age-related vascular stiffening: causes and consequences. Front Genet. 2015;6:112.

31. O'Rourke MF, Hashimoto J. Mechanical factors in arterial aging: a clinical perspective. J Am Coll Cardiol. 2007;50(1):1-13.

32. Safar ME, et al. Obesity, arterial stiffness, and cardiovascular risk. J Am Soc Nephrol. 2006;17(4 suppl 2):S109-S111.
33. Sethi S, et al. Aortic stiffness: pathophysiology, clinical implications, and approach to treatment. Integr Blood Press Control. 2014;7:29-34.

34. Fry JL, et al. Vascular smooth muscle sirtuin-1 protects against diet-induced aortic stiffness. Hypertension. 2016;68(3):775-784.

35. Weisbrod RM, et al. Arterial stiffening precedes systolic hypertension in diet-induced obesity. Hypertension. 2013;62(6):1105-1110.

36. Dolan E, et al. Ambulatory arterial stiffness index as a predictor of cardiovascular mortality in the Dublin Outcome Study. Hypertension. 2006;47(3):365-370.

37. Mattace-Raso FU, et al. Arterial stiffness and risk of coronary heart disease and stroke: the Rotterdam Study. Circulation. 2006;113(5):657-663.

38. Santhanam L, et al. Arginase and vascular aging. JAppl Physiol (1985). 2008;105(5):1632-1642.

39. Steppan J, et al. Exercise, vascular stiffness, and tissue transglutaminase. J Am Heart Assoc. 2014;3(2):e000599.

40. Avolio A. Arterial stiffness. Pulse (Basel). 2013;1(1):14-28.

41. Butlin $\mathrm{M}$, et al. Measuring arterial stiffness in animal experimental studies. Arterioscler Thromb Vasc Biol. 2020;40(5):1068-1077.

42. Spagnoli LG, et al. Aging and atherosclerosis in the rabbit. 1. Distribution, prevalence and morphology of atherosclerotic lesions. Atherosclerosis. 1991;89(1):11-24.

43. North BJ, Sinclair DA. The intersection between aging and cardiovascular disease. Circ Res. 2012;110(8):1097-1108.

44. Tesauro M, et al. Arterial ageing: from endothelial dysfunction to vascular calcification. J Intern Med. 2017;281(5):471-482.

45. Camici GG, et al. Molecular mechanism of endothelial and vascular aging: implications for cardiovascular disease. Eur Heart J. 2015;36(48):3392-3403.

46. Mistriotis P, Andreadis ST. Vascular aging: molecular mechanisms and potential treatments for vascular rejuvenation. Ageing Res Rev. 2017;37:94-116.

47. Kida Y, Goligorsky MS. Sirtuins, cell senescence, and vascular aging. Can J Cardiol. 2016;32(5):634-641.

48. Lacolley P, et al. Vascular smooth muscle cells and arterial stiffening: relevance in development, aging, and disease. Physiol Rev. 2017;97(4):1555-1617.

49. Jaminon A, et al. The role of vascular smooth muscle cells in arterial remodeling: focus on calcification-related processes. Int J Mol Sci. 
2019;20(22):5694.

50. Andrae J, et al. Role of platelet-derived growth factors in physiology and medicine. Genes Dev. 2008;22(10):1276-1312.

51. Gerthoffer WT. Mechanisms of vascular smooth muscle cell migration. Circ Res. 2007;100(5):607-621.

52. Raines EW. PDGF and cardiovascular disease. Cytokine Growth Factor Rev. 2004;15(4):237-254.

53. Hu W, Huang Y. Targeting the platelet-derived growth factor signalling in cardiovascular disease. Clin Exp Pharmacol Physiol. 2015;42(12):1221-1224.

54. Yang XP, et al. Making up or breaking up: the tortuous role of platelet-derived growth factor in vascular ageing. Clin Exp Pharmacol Physiol. 2009;36(8):739-747.

55. Ouyang L, et al. Roles of platelet-derived growth factor in vascular calcification. J Cell Physiol. 2018;233(4):2804-2814.

56. Kida T, et al. Chronic treatment with PDGF-BB and endothelin-1 synergistically induces vascular hyperplasia and loss of contractility in organ-cultured rat tail artery. Atherosclerosis. 2011;214(2):288-294.

57. Vazquez-Padron RI, et al. Aging exacerbates neointimal formation, and increases proliferation and reduces susceptibility to apoptosis of vascular smooth muscle cells in mice. J Vasc Surg. 2004;40(6):1199-1207.

58 . Rossi $\mathrm{E}$, et al. Increased plasma levels of platelet-derived growth factor (PDGF-BB + PDGF-AB) in patients with never-treated mild essential hypertension. Am J Hypertens. 1998;11(10):1239-1243.

59. Bath PM, Martin JF. Serum platelet-derived growth factor and endothelin concentrations in human hypercholesterolaemia. J Intern Med. 1991;230(4):313-317.

60. Wang L, et al. Oxidized phospholipids are ligands for LRP6. Bone Res. 2018;6:22.

61. Martinez-Lemus LA, et al. Amiloride improves endothelial function and reduces vascular stiffness in female mice fed a western diet. Front Physiol. 2017;8:456.

62. Jia G, et al. Endothelial mineralocorticoid receptor mediates diet-induced aortic stiffness in females. Circ Res. 2016;118(6):935-943.

63. Bonner JC. Regulation of PDGF and its receptors in fibrotic diseases. Cytokine Growth Factor Rev. 2004;15(4):255-273.

64. Ikura Y, et al. Expression of platelet-derived growth factor and its receptor in livers of patients with chronic liver disease. J Gastroenterol. 1997;32(4):496-501.

65. Gay S, et al. Immunohistologic demonstration of platelet-derived growth factor (PDGF) and sis-oncogene expression in scleroderma. J Invest Dermatol.1989;92(2):301-303.

66. He C, et al. PDGFR $\beta$ signalling regulates local inflammation and synergizes with hypercholesterolaemia to promote atherosclerosis. $\mathrm{Nat} \mathrm{Com-}$ mun. 2015;6:7770.

67. Brown XQ, et al. Effect of substrate stiffness and PDGF on the behavior of vascular smooth muscle cells: implications for atherosclerosis. J Cell Physiol. 2010;225(1):115-122.

68. Gallini R, et al. PDGF-A and PDGF-B induces cardiac fibrosis in transgenic mice. Exp Cell Res. 2016;349(2):282-290.

69. Qi YX, et al. PDGF-BB and TGF-\{beta\}1 on crosstalk between endothelial and smooth muscle cells in vascular remodeling induced by low shear stress. Proc Natl Acad Sci U S A. 2011;108(5):1908-1913.

70. Arita Y, et al. Adipocyte-derived plasma protein adiponectin acts as a platelet-derived growth factor-BB-binding protein and regulates growth factor-induced common postreceptor signal in vascular smooth muscle cell. Circulation. 2002;105(24):2893-2898.

71. Edwards JR, Mundy GR. Advances in osteoclast biology: old findings and new insights from mouse models. Nat Rev Rheumatol. 2011;7(4):235-243.

72. Park JH, et al. Current understanding of RANK signaling in osteoclast differentiation and maturation. Mol Cells. 2017;40(10):706-713.

73. Komutrattananont $\mathrm{P}$, et al. Morphology of the human aorta and age-related changes: anatomical facts. Anat Cell Biol. 2019;52(2):109-114.

74. Wheeler JB, et al. Relation of murine thoracic aortic structural and cellular changes with aging to passive and active mechanical properties. J Am Heart Assoc. 2015;4(3):e001744.

75. Berk BC, et al. Vasoconstriction: a new activity for platelet-derived growth factor. Science.
1986;232(4746):87-90.

76. Saltis J, et al. Age-dependent alterations in vascular smooth muscle cell responsiveness to platelet-derived growth factor in genetic hypertension. Clin Exp Pharmacol Physiol. 1993;20(5):324-326.

77. McCaffrey TA, et al. Aging and arteriosclerosis. The increased proliferation of arterial smooth muscle cells isolated from old rats is associated with increased platelet-derived growth factor-like activity. J Exp Med. 1988;167(1):163-174.

78. Wang M, et al. Proinflammatory profile within the grossly normal aged human aortic wall. Hypertension. 2007;50(1):219-227.

79. Steppan J, et al. Lysyl oxidase-like 2 depletion is protective in age-associated vascular stiffening. Am JPhysiol Heart Circ Physiol. 2019;317(1):H49-H59.

80. Maeda Y, et al. Enhanced contractile response of the basilar artery to platelet-derived growth factor in subarachnoid hemorrhage. Stroke. 2009;40(2):591-596.

81. Rieg AD, et al. PDGF-BB regulates the pulmonary vascular tone: impact of prostaglandins, calcium, MAPK- and PI3K/AKT/mTOR signalling and actin polymerisation in pulmonary veins of guinea pigs. Respir Res. 2018;19(1):120.

82. Leveen P, et al. Mice deficient for PDGF B show renal, cardiovascular, and hematological abnormalities. Genes Dev. 1994;8(16):1875-1887.

83. Zhang ZW, et al. Platelet-derived growth factor-induced severe and chronic vasoconstriction of cerebral arteries: proposed growth factor explanation of cerebral vasospasm. Neurosurgery. 2010;66(4):728-735.

84. Sarzani R, et al. Effects of hypertension and aging on platelet-derived growth factor and platelet-derived growth factor receptor expression in rat aorta and heart. Hypertension. 1991;18(5 suppl):III93-III99.

85. Liu X, et al. Osteoclasts protect bone blood vessels against senescence through the angiogenin/ plexin-B2 axis. Nat Commun. 2021;12(1):1832.

86. Li C, et al. Programmed cell senescence in skeleton during late puberty. Nat Commun. 2017;8(1):1312.

87. Zeadin M, et al. Effect of leptin on vascular calcification in apolipoprotein E-deficient mice. Arterio scler Thromb Vasc Biol. 2009;29(12):2069-2075. 